\title{
The Glimm scheme for perfect fluids on plane-symmetric Gowdy spacetimes
}

\author{
A P Barnes ${ }^{1}$, P G Lefloch ${ }^{2}$, B G Schmidt ${ }^{3}$ and J M Stewart ${ }^{1}$ \\ ${ }^{1}$ Department of Applied Mathematics and Theoretical Physics, Centre for Mathematical \\ Sciences, Cambridge CB3 0WA, UK \\ ${ }^{2}$ Laboratoire Jacques-Louis Lions \& Centre National de la Recherche Scientifique, UMR 7598, \\ Université Pierre et Marie Curie, BC 187, 75252 Paris Cedex 05, France \\ ${ }^{3}$ Max Planck Institut für Gravitationsphysik, Albert-Einstein-Institut, Am Mühlenberg 1, \\ D-14476 Golm, Germany \\ E-mail: a.p.barnes@damtp.cam.ac.uk,j.m.stewart@damtp.cam.ac.uk, \\ lefloch@cmap.polytechnique.fr and bernd.schmidt@aei.mpg.de
}

Received 31 August 2004

Published 20 October 2004

Online at stacks.iop.org/CQG/21/5043

doi:10.1088/0264-9381/21/22/003

\begin{abstract}
We propose a new, augmented formulation of the coupled Euler-Einstein equations for perfect fluids on plane-symmetric Gowdy spacetimes. The unknowns of the augmented system are the density and velocity of the fluid and the first- and second-order spacetime derivatives of the metric. We solve the Riemann problem for the augmented system, allowing propagating discontinuities in both the fluid variables and the first- and second-order derivatives of the geometry coefficients. Our main result, based on Glimm's random choice scheme, is the existence of solutions with bounded total variation of the Euler-Einstein equations, up to the first time where a blow-up singularity (unbounded first-order derivatives of the geometry coefficients) occurs. We demonstrate the relevance of the augmented system for numerical relativity. We also consider general vacuum spacetimes and solve a Riemann problem, by relying on a theorem by Rendall on the characteristic value problem for the Einstein equations.
\end{abstract}

PACS numbers: $04.20 . \mathrm{Cv}, 04.20 . \mathrm{Ex}, 04.30 .-\mathrm{w}, 47.75 .+\mathrm{f}$

\section{Introduction}

It is well known that solutions of the Euler equations for inviscid hydrodynamics can develop 'shocks' or discontinuities in the density and velocity, even starting from smooth initial data. The same is known to be true for special relativistic hydrodynamics assuming plane symmetry. What happens when general relativity is taken into account? A shock 
would imply a discontinuity in the energy-momentum tensor and hence, via Einstein's field equations, a discontinuity in the Ricci curvature tensor or, equivalently, jumps in the second derivatives of certain metric tensor components. But do Einstein's equations permit such discontinuities? If the sources and fields are weak so that linearized theory is applicable, it seems very plausible physically that they should. But what of the full nonlinear theory? Although there is a rich literature concerned with relativistic jump conditions (for instance [12]), the relativistic Rankine-Hugoniot relations, very little seems to have been done to establish existence and uniqueness of discontinuous solutions of the field equations.

The first rigorous result of which we are aware is due to Smoller and Temple [18]. They looked at the special relativistic Euler equations and assumed planar symmetry. (In Cartesian coordinates $(t, x, y, z)$ all quantities are assumed to depend on $t$ and $x$ only.) They demonstrated the existence of global solutions with bounded variation, by generalizing Nishida's proof $[13,14]$ for the non-relativistic system.

Much earlier Taub [19] had proposed an approximation scheme for solving the Einstein field equations with planar symmetry and not-so-weak fields. At the lowest level one has precisely the special relativity problem. Taub assumed the Smoller-Temple result and then described a systematic iteration process to include the matter-geometry and geometrygeometry interactions. A demonstration of its convergence would appear to be a daunting problem.

Rigorous existence results for full general relativity have been obtained by Groah and Temple $[6,7]$ under the assumption of spherical symmetry. While their results are very interesting we feel that the assumption of spherical symmetry is restrictive. We shall argue that general relativistic shocks are a fascinating example of the features of hyperbolic systems. But for spherical symmetry many of these features are suppressed (cf Birkhoff's theorem [21]).

There is an important difference between classical hydrodynamics and Einstein's theory. Reductions of Einstein's equations produce a hyperbolic system but with elliptic constraint equations. The latter are often regarded as secondary, subsidiary features but we shall argue that they are of pivotal importance. For a related discussion see, e.g., [4].

The use of the space of functions with bounded variation in general relativity is not new; cf the extensive and profound work by Christodoulou (for instance in [2]) on the Einstein equations coupled to a scalar field.

We start by considering the vacuum case for this is the key to the entire discussion. It is explored in detail in the next section. Within an initial spacelike hypersurface we assume that there is an internal hypersurface (i.e., a 2-surface) across which some second transverse derivatives of the 3-metric are discontinuous. (We do not ask how these discontinuities occurred.) By imposing the constraint equations we determine directly which discontinuities are permitted. We next demonstrate that for permissible discontinuities we can solve the initial data problem, i.e., determine the initial data for Einstein's equations on the initial hypersurface which satisfy the constraint equations. The Cauchy problem has initial data discontinuous across a 2-surface and so resembles a Riemann problem. On each side of the discontinuity we can solve a standard Cauchy problem within the appropriate domain of dependence. Reading off the solutions on the future boundary of each side we have sufficient data to solve the characteristic initial value problem to the future of the boundaries, according to a theorem of Rendall [15]. This produces an existence/uniqueness result which is local, i.e., valid in a small spacetime neighbourhood of the initial discontinuity. In each domain of dependence and in their common future the solution is smooth. However if there were discontinuities in the initial data then there will be discontinuities in the second transverse derivatives of the metric 
across the boundaries. Apart from vacuum, no other assumption is required, and so this is a general result.

We next turn to the case where an inviscid perfect fluid is present. In classical (nonrelativistic) hydrodynamics, existence/uniqueness theorems are usually based on a technique introduced by Glimm [5] (cf [11] for recent developments) which assumes planar symmetry. We therefore restrict consideration to a subclass of plane-symmetric Gowdy spacetimes whose general properties are described in sections 3 and 4 . In section 5 we introduce the new, augmented formulation in which the first- and second-order derivatives of the metric components are considered as independent unknowns. Section 6 states precisely the main result of this paper, an existence theorem for arbitrary large initial data. We write the field equations as a quasilinear system of conservation laws with sources. This differs from standard reductions in that we write down equations for the evolution of first and second derivatives of metric coefficients, allowing them to be as discontinuous as the fluid variables. Further we assume that the initial data satisfy the constraints for we do not use the constraint equations directly in our analysis. We treat the convection terms through a Riemann problem defined in section 7. We introduce a generalization to the Glimm scheme, whose convergence is established in section 8. The main difficulties for the convergence analysis are to ensure that (1) the total variation of the fluid and the first-order derivatives of the geometry coefficients have uniformly bounded variation, and that (2) the energy density remains bounded away from zero and the velocity remains bounded away from the light speed. This leads us to the main result of this paper, the existence theorem stated in section 6 .

In section 9 we describe the numerical implementation of a finite difference scheme based on the augmented formulation, and show some results from numerical simulations. A final section provides some concluding observations.

\section{The vacuum case}

Let $\Sigma$ be a spacelike hypersurface in spacetime $\mathcal{M}$ with intrinsic curvature tensors $h_{a b}$ and $k_{a b}$. Suppose there is a surface $S \subset \Sigma$ such that

(1) the fields $h_{a b}$ and $k_{a b}$ are $C^{\infty}$ on the complement of $S$,

(2) the second transverse derivatives may be discontinuous across $S$.

We first ask what restrictions are imposed on such jumps by the constraint equations, then whether the constraint equations can be solved, and finally whether the vacuum Einstein field equations can be solved. For the notation used in this section we refer to the textbook [21].

The constraint equations are

$$
\begin{aligned}
& k_{a}{ }^{b}{ }_{\mid b}-k_{, a}=0, \\
& { }^{3} R+k_{a b} k^{a b}-k^{2}=0 .
\end{aligned}
$$

Here denotes the covariant derivative of $h_{a b},{ }^{3} R$ its Ricci scalar and $k=k_{a}{ }^{a}$. Since the questions we are asking are local we use Gaussian normal coordinates adapted to $S$ so that $S$ is $\left(x^{1}=0\right)$ and

$$
\mathrm{d} s^{2}=\mathrm{d} x^{1^{2}}+h_{A B} \mathrm{~d} x^{A} \mathrm{~d} x^{B}
$$

where $A, B, \ldots$ range over 2,3 . We are interested in the second transverse derivatives of $h_{A B}$ and the first transverse derivatives of $k_{A B}$. We may decompose the momentum constraint as

$$
k_{1}{ }^{1}{ }_{, 1}+k_{1}{ }^{A}{ }_{, A}-k_{a}{ }^{a}{ }_{, 1}+\cdots=0, \quad k_{A}{ }^{1}{ }_{, 1}+k_{A}{ }^{B}{ }_{, B}-k_{a}{ }^{a}{ }_{, A}+\cdots=0,
$$


where $\cdots$ denotes terms containing no derivatives of $k_{a b}$ and at most first derivatives of $h_{a b}$ so that they are continuous at $S$. We deduce immediately that $k_{A 1,1}$ and $k_{A}{ }^{A}{ }_{, 1}$ must be continuous across $S$.

Next we introduce the notation $d_{A B}=-\frac{1}{2} h_{A B, 1}$ and $d=h^{A B} d_{A B}$. Then the Hamiltonian constraint can be written in the form

$$
{ }^{2} R-h^{A B} d_{A B, 1}+d^{2}+3 d_{A B} d^{A B}+k_{a b} k^{a b}-k^{2}=0,
$$

where ${ }^{2} R$ is the Ricci scalar curvature of $h_{A B}$. Now $h^{A B} d_{A B, 1}=d_{, 1}+2 d_{A B} d^{A B}$ and so we see that $d_{, 1}$ must be continuous. Thus we can allow jumps only in the trace-free parts of $d_{A B, 1}$ and $k_{A B, 1}$. If we introduce the normal (in $\mathcal{M}$ ) to the surface $\Sigma$ and decompose the Weyl tensor into its electric and magnetic parts then only the trace-free parts of these can have jumps. Equivalently if we introduce a Newman-Penrose null tetrad with $l^{\mu}, n^{\mu}$ spanning the 2-space generated by the normal and $\partial / \partial x^{1}$ then $\Psi_{1}, \Psi_{2}$ and $\Psi_{3}$ must be continuous across $S$; only $\Psi_{0}$ and $\Psi_{4}$ can have jumps.

Can we find solutions of the constraint equations with these properties? Consider first the case of time-symmetric data, $k_{a b}=0$. Let $\widetilde{h}_{A B}$ be any symmetric tensor field such that the trace-free part of $\widetilde{h}_{A B, 11}$ is discontinuous and require that $f\left(x^{a}\right) \widetilde{h}_{A B}$ satisfies the Hamiltonian constraint. The equation for $f$ is

$$
\Delta f-\frac{1}{6}{ }^{3} \widetilde{R} f=0,
$$

where ${ }^{3} \widetilde{R}$ is the Ricci scalar of $\widetilde{h}_{A B}$. We can solve this easily in two special cases:

(1) If $\widetilde{h}_{A B}$ is piecewise analytic we can impose boundary data

$$
f\left(0, x^{A}\right)=1, \quad f_{, 1}\left(0, x^{A}\right)=0
$$

and use the Cauchy-Kovalevski theorem.

(2) If we set $\widetilde{h}_{A B}=\widetilde{h}_{A B}\left(x^{1}\right)$ and $f=f\left(x^{1}\right)$ then we have an ordinary differential equation which can be solved. (Such data admit an Abelian two-dimensional isometry group.)

In the general case we have to try a different approach. We prescribe $h_{A B}$ arbitrarily and use the constraint equations to obtain parts of $k_{a b}$. We decompose $k_{a}{ }^{b}$ into $k_{1}{ }^{1}, k_{1}{ }^{B}, k_{A}{ }^{B}$ and further decompose $k_{A}{ }^{B}$ into ${ }^{2} k$ (its trace) and $\widetilde{k}_{A}^{B}$ (its tracefree part). We prescribe $\widetilde{k}_{A}^{B}$ with a jump in $S$. In the Hamiltonian constraint $k_{1}{ }^{1}$ appears only in the form $k_{1}{ }^{1}\left(k_{2}{ }^{2}+k_{3}{ }^{3}\right)$ and we can solve for it provided ${ }^{2} k \neq 0$. In the momentum constraint the $k_{1}{ }^{1}, 1$ terms cancel and we can solve for ${ }^{2} k_{, 1}$ and $k_{1}{ }^{A}, 1$. Assuming again analyticity or symmetry we can obtain a solution if we give initial values ${ }^{2} k \neq 0$ and ${k_{1}}^{A}$ at $S$.

We next introduce a chart in spacetime $\mathcal{M}$ as follows. (Figure 1 may be helpful here.) $N_{0}$ and $N_{1}$ are null hypersurfaces in $\mathcal{M}$ which intersect in $S . x^{0}$ is an affine parameter along the null geodesic generators of $N_{0}$ such that $x^{0}=0$ at $S$. It is also defined to be zero on $N_{1} \cdot x^{1}$ is similarly defined, so that $S$ is $x^{0}=x^{1}=0 .\left(x^{A}\right)$ are the intrinsic coordinates in $S$ and we propagate them onto $N_{0}$ and $N_{1}$ by requiring them to be constant along the null generators. This defines $\left(x^{\alpha}\right)$ on $N_{0} \cup N_{1}$. We propagate them into the future $U$ by requiring $\square x^{a}=0$ and solving the characteristic initial value problem.

We relabel the initial data on $\Sigma$ on each side of $S$ as ${ }^{0} h_{a b}$ and ${ }^{1} h_{a b}$ and extend each of them smoothly across $S$ in an arbitrary way. Consider first the data ${ }^{0} h_{a b}$. By solving the standard Cauchy problem we can determine, at least locally, a smooth solution in the domain of dependence $U_{0}$. Since this includes $N_{0}$ we can obtain the restriction of ${ }^{0} g_{A B}$ to $N_{0}$ by taking the limit from the past. The same can be done on $N_{1}$. Then a theorem of Rendall [15] guarantees the local existence and uniqueness of the solution of the characteristic initial value problem for the Einstein vacuum field equations in $U$. The solutions will be smooth in 


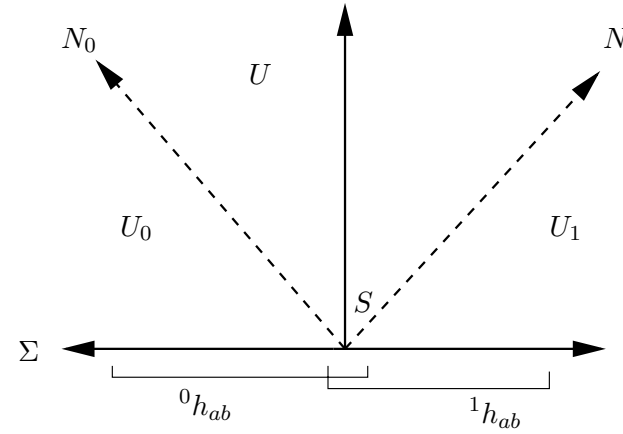

Figure 1. Spacetime $\mathcal{M}$ with two dimensions suppressed. Smooth data ${ }^{0} h_{a b}$ and ${ }^{1} h_{a b}$ are specified on the indicated regions of the Cauchy surface $\Sigma$, but they do not agree in the overlap at $S .{ }^{0} h_{a b}$ is used to determine the solution in the region $U_{0}$ and hence, by continuation from below, on the null hypersurface $N_{0}$. The data on the null hypersurfaces are used to solve a characteristic initial value problem for the metric in $U$. The second transverse derivatives of this solution on $N_{0}$ and $N_{1}$ are discontinuous.

$U_{0}, U_{1}$ and $U$, and across $N_{0}$ and $N_{1}$ they will be continuous with continuous transverse first derivatives, but the second transverse derivatives will have jumps.

Thus in the vacuum case we have a fairly complete local result.

\section{Plane-symmetric perfect fluids}

Even in the Newtonian case existence results are only known for the case of one spatial independent variable. Therefore we restrict consideration to plane-symmetric spacetimes.

We consider solutions of the Einstein-Euler equations for a compressible perfect fluid, assuming that the spacetime metric has the following 'polarized Gowdy' form,

$$
\mathrm{d} s^{2}=g_{\alpha \beta} \mathrm{d} x^{\alpha} \mathrm{d} x^{\beta}=\mathrm{e}^{2 a}\left(-\mathrm{d} t^{2}+\mathrm{d} x^{2}\right)+\mathrm{e}^{2 b}\left(\mathrm{e}^{2 c} \mathrm{~d} y^{2}+\mathrm{e}^{-2 c} \mathrm{~d} z^{2}\right)
$$

where the coefficients $a, b, c$ only depend on the time variable $t$ and the space variable $x$, but are independent of $y$ and $z$. We consider perfect fluids with mass density $\rho>0$, energy density $\mu>0$ and pressure $p$. These thermodynamical variables are connected via the so-called equation of state of the fluid: $p=p(\rho, \mu)$. For simplicity (we do not have to worry about the evolution of $\rho$ ) we consider an ultrarelativistic fluid whose equation of state is

$$
p=c_{s}^{2} \mu, \quad 0<c_{s}<1,
$$

where the constant $c_{s}$ is called the sound speed.

The 4-velocity vector $u^{\alpha}$ is normalized to be of unit length

$$
u^{\alpha} u_{\alpha}=-1 \text {. }
$$

We make the simplifying assumption that the velocity is only in the $x$-direction, and set

$$
u^{\alpha}=\mathrm{e}^{-a} \gamma(1, v, 0,0),
$$

where the coordinate velocity $v$ satisfies $|v|<1$ and $\gamma=\left(1-v^{2}\right)^{-1 / 2}$. The matter is described by the energy-momentum tensor

$$
T^{\alpha \beta}=(\mu+p) u^{\alpha} u^{\beta}+p g^{\alpha \beta},
$$

from which we extract fields $\tau, S$ and $\Sigma$ defined via

$$
T^{00}=\mathrm{e}^{-2 a} \tau=\mathrm{e}^{-2 a}\left((\mu+p) \gamma^{2}-p\right),
$$




$$
T^{01}=\mathrm{e}^{-2 a} S=\mathrm{e}^{-2 a}(\mu+p) \gamma^{2} v
$$

and

$$
T^{11}=\mathrm{e}^{-2 a} \Sigma=\mathrm{e}^{-2 a}\left((\mu+p) \gamma^{2} v^{2}+p\right) .
$$

Note that given $\mu, v$ and $p$, which we will consider as our primary unknowns, or primitive variables, it is easy to compute $\tau, S$ and $\Sigma$, which we shall call conserved variables. Given the conserved variables $\tau$ and $S$ it is straightforward to compute primitive variables $\mu$ and $v$ and hence $p$. These calculations take place in Minkowski spacetime, which is independent of the geometry variables $a, b$ and $c$.

Because $p=c_{s}^{2} \mu$, we have

$$
\begin{aligned}
& \tau=(\mu+p) \gamma^{2}-p=\left(\left(1+c_{s}^{2}\right) \gamma^{2}-c_{s}^{2}\right) \mu, \\
& S=(\mu+p) \gamma^{2} v=\left(1+c_{s}^{2}\right) \gamma^{2} v \mu, \\
& \Sigma=(\mu+p) \gamma^{2} v^{2}+p=\left(\left(1+c_{s}^{2}\right) \gamma^{2} v^{2}+c_{s}^{2}\right) \mu,
\end{aligned}
$$

and it is convenient to note here that

$$
-\tau+\Sigma-2 p=-\left(1+c_{s}^{2}\right) \mu, \quad \tau-\Sigma=\left(1-c_{s}^{2}\right) \mu .
$$

The Einstein field equations are

$$
G^{\alpha \beta}=\kappa T^{\alpha \beta},
$$

where $G^{\alpha \beta}$ is the Einstein tensor and $\kappa=8 \pi G / c^{4}$. Here $G$ is Newton's gravitational constant, and $c$ is the speed of light which (by appropriate choice of physical units) is set to unity throughout this paper.

Using the line element (3.1) we may compute the constraint equations, the (00) and (01) components of (3.8) as

$$
\begin{aligned}
& 2 a_{t} b_{t}+2 a_{x} b_{x}+b_{t}^{2}-2 b_{x x}-3 b_{x}^{2}-c_{t}^{2}-c_{x}^{2}=\kappa \mathrm{e}^{2 a} \tau, \\
& -2 a_{t} b_{x}-2 a_{x} b_{t}+2 b_{t x}+2 b_{t} b_{x}+2 c_{t} c_{x}=\kappa \mathrm{e}^{2 a} S,
\end{aligned}
$$

as well as the evolution equations, the (11), (22) and (33) components

$2 a_{t} b_{t}+2 a_{x} b_{x}-2 b_{t t}-3 b_{t}^{2}+b_{x}^{2}-c_{t}^{2}-c_{x}^{2}=\kappa \mathrm{e}^{2 a} \Sigma$,

$-a_{t t}+a_{x x}-b_{t t}-b_{t}^{2}+2 b_{t} c_{t}+b_{x x}+b_{x}^{2}-2 b_{x} c_{x}+c_{t t}-c_{t}^{2}-c_{x x}+c_{x}^{2}=\kappa \mathrm{e}^{2 a} p$,

and

$-a_{t t}+a_{x x}-b_{t t}-b_{t}^{2}-2 b_{t} c_{t}+b_{x x}+b_{x}^{2}+2 b_{x} c_{x}-c_{t t}-c_{t}^{2}+c_{x x}+c_{x}^{2}=\kappa \mathrm{e}^{2 a} p$.

The evolution equations contain second time derivatives of $a, b$ and $c$, whereas the constraint equations contain only zero or first time derivatives.

It is convenient to take certain linear combinations: computing ' $-(3.9)+(3.11)-(3.12)-$ (3.13)' gives

$$
a_{t t}-a_{x x}=b_{t}^{2}-b_{x}^{2}-c_{t}^{2}+c_{x}^{2}+\frac{1}{2} \kappa \mathrm{e}^{2 a}(-\tau+\Sigma-2 p),
$$

while '(3.9) - (3.11)' yields

$$
b_{t t}-b_{x x}=-2 b_{t}^{2}+2 b_{x}^{2}+\frac{1}{2} \kappa \mathrm{e}^{2 a}(\tau-\Sigma),
$$

and finally '(3.12) - (3.13)' simplifies to

$$
c_{t t}-c_{x x}=-2 b_{t} c_{t}+2 b_{x} c_{x} .
$$


The evolution equations for the fluid are a consequence of the Einstein field equations (3.8) and are obtained by expressing the Bianchi identities $G^{\alpha \beta} ; \beta=0$ for the metric in terms of the energy-momentum tensor

$$
T_{; \beta}^{\alpha \beta}=0,
$$

where $; \beta$ denotes the covariant derivative. We thus obtain the Euler equations

$$
\tau_{t}+S_{x}=T_{1}, \quad S_{t}+\Sigma_{x}=T_{2},
$$

in which the 'source terms' $T_{1}$ and $T_{2}$ are given by

$$
\begin{aligned}
& T_{1}=-\tau\left(a_{t}+2 b_{t}\right)-S\left(2 a_{x}+2 b_{x}\right)-\Sigma a_{t}-2 p b_{t}, \\
& T_{2}=-\tau a_{x}-S\left(2 a_{t}+2 b_{t}\right)-\Sigma\left(a_{x}+2 b_{x}\right)+2 p b_{x} .
\end{aligned}
$$

Note that if we set $a \equiv b \equiv c \equiv 0$, forcing the spacetime to be flat then the right-hand sides of (3.18) are zero. In this case we have the special relativistic Euler equations.

Next we examine the constraint equations (3.9) and (3.10). It is convenient to define

$$
\mathcal{H}=\mathrm{e}^{2 b}\left(2 a_{t} b_{t}+2 a_{x} b_{x}+b_{t}^{2}-2 b_{x x}-3 b_{x}^{2}-c_{t}^{2}-c_{x}^{2}-\kappa \mathrm{e}^{2 a} \tau\right),
$$

and

$$
\mathcal{M}=\mathrm{e}^{2 b}\left(-2 a_{t} b_{x}-2 a_{x} b_{t}+2 b_{t x}+2 b_{t} b_{x}+2 c_{t} c_{x}-\kappa \mathrm{e}^{2 a} S\right),
$$

so that the constraint equations are equivalent to $\mathcal{H}=\mathcal{M}=0$. If we form the time derivatives of $\mathcal{H}$ and $\mathcal{M}$ and make use of the evolution equations we obtain

$$
\mathcal{H}_{t}+\mathcal{M}_{x}=0=\mathcal{M}_{t}+\mathcal{H}_{x},
$$

a symmetric hyperbolic system of conservation laws (with characteristic velocities \pm 1 ) for $\mathcal{H}$ and $\mathcal{M}$. Thus if the conservation equations are satisfied at say $t=0$ for all $x$ and the evolution equations (3.14)-(3.18) are satisfied for $t \geqslant 0$ and all $x$, then the constraint equations are satisfied for $t \geqslant 0$ and all $x$.

This completes the description of the system under study.

Remark 3.1. It should be noted that the limiting case $c_{s}=1$ is very different. For $c_{s}<1$ and the energy momentum tensor (3.2), the conservation equations (3.17) imply the nonlinear Euler equations. However if $c_{s}=1$ it is easy to verify that the solution of (3.17) is

$$
\mu=\left(\nabla_{\beta} \phi\right)\left(\nabla^{\beta} \phi\right), \quad u^{\alpha}=\mu^{-1 / 2} \nabla^{\alpha} \phi,
$$

where $\phi$ satisfies the linear wave equation

$$
g^{\alpha \beta} \nabla_{\alpha} \nabla_{\beta} \phi=0 .
$$

\section{Special solutions}

Before we present the main statement in this paper (section 5), it is useful to investigate the existence and properties of special solutions, and to discuss the general algebraic properties of the system under consideration. One important issue of interest before we can develop a theory of shock waves is to determine the functional space suited to our problem.

We begin by formally decoupling the fluid and the metric and considering the Einstein equations in vacuum for metric (3.1). Thus we consider the following equations for the variables $a, b, c$ :

$$
a_{t t}-a_{x x}=b_{t}^{2}-b_{x}^{2}-c_{t}^{2}+c_{x}^{2},
$$




$$
b_{t t}-b_{x x}=-2 b_{t}^{2}+2 b_{x}^{2}
$$

and

$$
c_{t t}-c_{x x}=-2 b_{t} c_{t}+2 b_{x} c_{x} .
$$

In addition we must satisfy the constraint equations

$$
2 a_{t} b_{t}+2 a_{x} b_{x}+b_{t}^{2}-2 b_{x x}-3 b_{x}^{2}-c_{t}^{2}-c_{x}^{2}=0,
$$

and

$$
-2 a_{t} b_{x}-2 a_{x} b_{t}+2 b_{t x}+2 b_{t} b_{x}+2 c_{t} c_{x}=0
$$

Theorem 4.1. When the coupling with the fluid is neglected (that is, by formally replacing the coupling constant $\kappa$ by 0 ), the geometric variables $a, b, c$ may blow up in amplitude in finite time. More precisely, the following hold:

- The general solution $b=b(t, x)$ of (4.2) may exhibit blow up in finite time.

- As long as b remains bounded, the variables a and c remain bounded as well.

Furthermore, the initial value problem for the vacuum polarized Gowdy equations admits a smooth, globally defined solution (for all $t \geqslant 0$ and all $x$ ) when the initial data for $a, b, c$ are smooth compactly supported functions and the initial data for $b$ are sufficiently small, in the sense that

$$
\int_{\mathbb{R}} \mathrm{e}^{2 b(0, x)}\left(\left|b_{x}(0, x)\right|+\left|b_{t}(0, x)\right|\right) \mathrm{d} x \ll 1 .
$$

Therefore, the function $b$ determines whether there are singularities in the geometric variables. A blow up of $b$ corresponds to the formation of a singularity in the metric. Theorem 4.1 explains why the existence result in section 5 can cover only a finite time interval for general data.

Proof. We observe that the nonlinear equation (4.2) for the function $b$ is equivalent to the linear wave equation

$$
\left(\mathrm{e}^{2 b}\right)_{t t}-\left(\mathrm{e}^{2 b}\right)_{x x}=0
$$

and can therefore be integrated explicitly:

$$
b(x, t)=\frac{1}{2} \log (f(x+t)+g(x-t)), \quad b_{x}(x, t)=\frac{1}{2} \frac{f^{\prime}(x+t)+g^{\prime}(x-t)}{f(x+t)+g(x-t)},
$$

where $f$ and $g$ are arbitrary functions satisfying $f+g>0$. The function $\mathrm{e}^{2 b}$ being the solution of a linear wave equation, may well be positive initially but it can vanish after a finite time, corresponding to a blow up of the coefficient $b$ itself. Clearly, depending upon the choice of the functions $f$ and $g$, the function $b$ and its derivatives may (or may not) blow up in finite time. However, when $f$ and $g$ are sufficiently close to a positive constant, or equivalently when $b_{x}$, and $b_{t}$ are sufficiently small at time $t=0$, then the solution $b$ remains close to 0 for all times and no blow up takes place.

If $b$ is already determined and smooth, equation (4.3) can be regarded as a linear equation in $c$ and therefore its solutions cannot blow up in finite time. Similarly, if both $b$ and $c$ are already determined, the coefficient $a$ is the solution of a linear wave equation (4.1) with given right-hand side. Again no blow up can possibly take place.

A special class of homogeneous vacuum solutions, possibly exhibiting blow up, is obtained by assuming that $a, b, c$ are independent of the space variable. Note that $b$ does not have compact support and, therefore, the following result does not contradict the global existence result in theorem 4.1 . 
Theorem 4.2. When the coupling with the fluid is neglected, the homogeneous solutions have the form

$$
\mathrm{e}^{2 a}=A\left|t-t_{0}\right|^{2 k^{2}-1 / 2}, \quad \mathrm{e}^{2 b}=B\left|t-t_{0}\right|, \quad \mathrm{e}^{2 c}=C\left|t-t_{0}\right|^{2 k}
$$

where $t_{0}, A, B, C$ and $k$ are constant parameters. The curvature invariant $R^{\alpha \beta \gamma \delta} R_{\alpha \beta \gamma \delta}$ is proportional to $\left|t-t_{0}\right|^{-4 k^{2}-3}$. Thus by suitable choice of the parameters we can arrange blow up at $t=t_{0}$ in the future.

We now turn to the coupled Einstein-Euler system. Here there are a few exact solutions, including a homogeneous one [1]. Let $\alpha$ and $m$ be positive constants, and set

$$
f(s)=\left(\alpha+m s^{1-c_{s}^{2}}\right)^{1 /\left(1-c_{s}^{2}\right)} .
$$

Define $\tau$ implicitly via

$$
t=\int_{0}^{\tau} \frac{s^{1 / 3}}{(f(s))^{1-c_{s}{ }^{2}}} \mathrm{~d} s .
$$

Then the solution is given by

$$
\begin{array}{ll}
a & =-\frac{1}{3} \log \tau+\left(1-c_{s}^{2}\right) \log f(\tau), \quad b=\frac{2}{3} \log \tau, \quad c=0 \\
\mu & =\frac{4 m}{3(\tau f(\tau))^{1+c_{s}^{2}}}, \quad v=0 .
\end{array}
$$

The singularity here occurs at $t=\tau=0$. By choosing data at some positive $t$ and timereversing it we can arrange for blow up in the future. For a related discussion see Rendall [16].

\section{The augmented Euler-Einstein system}

Consider the two constraint equations (3.9), (3.10). It is natural to place the terms $b_{x x}$ and $b_{t x}$ (which have the highest derivatives) in the same functional class as the unknowns $\tau$ and $S$ (which may have jumps). This motivates us to consider $b_{x x}$ and $b_{t x}$ as independent variables, and, in order to treat all geometry variables on the same level of regularity, we set

$U:=\left(a_{t x}, a_{x x}, b_{t x}, b_{x x}, c_{t x}, c_{x x},\right), \quad V:=(\tau, S), \quad W:=\left(\mathrm{e}^{2 a}, a_{t}, a_{x}, b_{t}, b_{x}, c_{t}, c_{x}\right)$.

The augmented system associated with the equations under consideration has the abstract form

$U_{t}+A^{1} U_{x}+A^{2} V_{x}+A^{3} W_{x}=R(U, W), \quad U=U(t, x) \in \mathbb{R}^{6}$,

$V_{t}+B(V) V_{x}=S(V, W), \quad V=V(t, x) \in \mathbb{R}_{+} \times \mathbb{R}$,

$W_{t}+C W_{x}=T(V, W)$,

$W=W(t, x) \in \mathbb{R}_{+} \times \mathbb{R}^{6}$.

Specifically, we find

$U_{1 t}-U_{2 x}+\frac{1}{2} \kappa\left(1+c_{s}^{2}\right)\left(W_{1} \mu\right)_{x}=2\left(U_{3} W_{4}-U_{4} W_{5}-U_{5} W_{6}+U_{6} W_{7}\right)$,

$U_{2 t}-U_{3 x}=0$,

$U_{3 t}-U_{4 x}-\frac{1}{2} \kappa\left(1-c_{s}^{2}\right)\left(W_{1} \mu\right)_{x}=2\left(U_{3} W_{5}+U_{4} W_{6}\right)$,

$U_{4 t}-U_{3 x}=0$, 
in which $\mu$ is a function of $V$, and

$$
\begin{aligned}
& U_{5 t}-U_{6 x}=2\left(-U_{3} W_{6}+U_{4} W_{7}-U_{5} W_{4}+U_{6} W_{5}\right), \\
& U_{6 t}-U_{5 x}=0 .
\end{aligned}
$$

The evolution equations for the $V$-variables are (3.18), that is with the new notation

$$
\begin{aligned}
& V_{1 t}+V_{2 x}=-V_{1}\left(W_{2}+2 W_{4}\right)-V_{2}\left(2 W_{3}+2 W_{5}\right)-\Sigma W_{2}-2 p W_{4}, \\
& V_{2 t}+\Sigma_{x}=-V_{1} W_{3}-V_{2}\left(2 W_{2}+2 W_{4}\right)-\Sigma\left(W_{3}+2 W_{5}\right)+2 p W_{5},
\end{aligned}
$$

in which $\Sigma, p$ are functions of $V$. Finally, for the variable $W$ we add

$$
\begin{aligned}
& W_{1 t}=2 W_{1} W_{2}, \\
& W_{2 t}-W_{3 x}=W_{4}^{2}-W_{5}^{2}-W_{6}^{2}-\frac{1}{2} \kappa W_{1}\left(1+c_{s}^{2}\right) \mu, \\
& W_{3 t}-W_{2 x}=0 \\
& W_{4 t}-W_{5 x}=-2 W_{4}^{2}+2 W_{5}^{2}+\frac{1}{2} \kappa W_{1}\left(1-c_{s}^{2}\right) \mu, \\
& W_{5 t}-W_{4 x}=0 \\
& W_{6 t}-W_{7 x}=-2 W_{4} W_{6}+2 W_{5} W_{7} \\
& W_{7 t}-W_{6 x}=0 .
\end{aligned}
$$

In addition, the system of evolution equations is supplemented with two constraints of the form

where $D$ and $E$ are given by

$$
D(U, V, W)=0, \quad E(U, V, W)=0,
$$

$$
\begin{aligned}
& 2 W_{2} W_{4}+2 W_{3} W_{5}+W_{4}^{2}-2 U_{4}-3 W_{5}^{2}-W_{6}^{2}-W_{7}^{2}=\kappa W_{1} V_{1}, \\
& -2 W_{2} W_{5}-2 W_{3} W_{4}+2 U_{3}+2 W_{4} W_{5}+2 W_{6} W_{7}=\kappa W_{1} V_{2} .
\end{aligned}
$$

If these constraints are satisfied at the initial time, then they are satisfied for all times as a consequence of the evolution equations. For suppose that $U, V$ and $W$ satisfy the evolution equations (5.1). It follows from the Bianchi identities that $D$ and $E$ satisfy a similar system with fluxes and sources which are homogeneous functions of $D$ and $E$ of degree 1 . Thus if $D$ and $E$ are zero initially they will remain so.

It is illuminating to compare the vacuum and fluid cases at this point. If the fluid has a shock then $V_{1}=\tau$ and $V_{2}=S$ will be discontinuous and so $U_{3}=b_{t x}$ and $U_{4}=b_{x x}$ might also be discontinuous, as a consequence of the constraint equations (5.8), (5.9) (assuming a priori that $W_{1}=\mathrm{e}^{a}$ is continuous). But in vacuum the same argument shows that these second derivatives of $b$ will be continuous. This is fully consistent with section 2 for trace $\left(h_{A B}\right)=\mathrm{e}^{4 b}$. In the vacuum case it is $c_{t x}$ and $c_{x x}$ which can carry discontinuities.

We observe that the matrix $A^{1}$ has constant coefficients and eigenvalues \pm 1 (each with multiplicity 3) and a full basis of eigenvectors. Basically, the unknown $U \in \mathbb{R}^{6}$ satisfies three wave equations in flat space, coupled only through the source term $R(U, W)$, a given algebraic expression of $U$ and $W$. Note however that the term $\left(W_{1} \mu\right)_{x}$ generates some additional coupling, that is the terms $A^{2} V_{x}+A^{3} W_{x}$, between the fluid and the geometry (which should not be handled as a source term since $\mu$ may contain discontinuities). To handle this coupling we will solve a Riemann problem (section 7).

On the other hand, the matrix $B(V)$ depends nonlinearly on $V$ and describes the standard relativistic fluid equations in a Minkowski background. The eigenvalues of the matrix $B(V)$ are those of the special relativistic Euler equations in flat space [18],

$$
\lambda_{ \pm}=\frac{v \pm c_{s}}{1 \pm v c_{s}} .
$$


Note that since $0<c_{s}<1, \lambda_{+}$is a strictly increasing function of $c_{s}$ and so $\lambda_{+}>\lambda_{-}$for all $v$ with $0 \leqslant|v|<1$.

Finally, we note that the matrix $C$ has constant coefficients and eigenvalues 0 and \pm 1 with multiplicities 0,3 and 3, and a full basis of eigenvectors. We have a simple evolution equation for $\mathrm{e}^{2 a}$ and three wave equations. As a matter of fact, the equations for $V$ and $W$ can be solved independently from the equations for $U$, and the latter serve only to propagate the constraints.

Remark 5.1. The limiting case $c_{s}=1$ needs special treatment. Note first that because of (3.7), the matter term on the right-hand side of (3.15) vanishes and so the $b$-equation reduces to its vacuum form (4.2) with explicit solution (4.6). The field equation (3.24) for $\phi$ implies

$$
\left(\mathrm{e}^{2 b} \phi_{t}\right)_{t}-\left(\mathrm{e}^{2 b} \phi_{r}\right)_{r}=0
$$

or

$$
\phi_{t t}-\phi_{x x}=-2 b_{t} \phi_{t}+2 b_{x} \phi_{x}
$$

which should be compared with the $c$-equation (4.3) in vacuum. Note also that (5.10) implies $\lambda_{ \pm}= \pm 1$.

\section{The Cauchy problem}

In this section we state the existence result to be established in this paper. With any function with bounded variation $Y: \mathbb{R} \rightarrow \mathbb{R}$ we associate its $L^{\infty}$ norm

$$
\|Y\|_{L^{\infty}}:=\sup _{x \in \mathbb{R}}|Y(x)|
$$

and its total variation

$$
T V(Y)=\sup \sum_{n}\left|Y\left(x_{n+1}\right)-Y\left(x_{n}\right)\right|<\infty,
$$

where the supremum is over all monotone increasing, finite sequences $x_{n}$. An alternative definition (which is essentially equivalent to the above) is to require

$$
\sup \left|\int_{\mathbb{R}} \theta_{x} Y \mathrm{~d} x\right|<\infty
$$

where the supremum is taken over all smooth functions with compact support satisfying $\|\theta\|_{L^{\infty}} \leqslant 1$. (In other words, a function has bounded variation if its derivative in the weak sense of distributions is a bounded measure.)

The relevance of the space of functions with bounded variation to study nonlinear hyperbolic systems was recognized by Glimm [5]. Recall that such a function $Y$ admits left-hand and right-hand traces $Y(x \pm)$ at each point $x$, which are distinct at countably many points, at most. This space is well suited to describe discontinuous solutions of hyperbolic equations. In addition, the analysis of approximate solutions is based on a key compactness theorem, due to Helly: if $Y^{h}$ is a sequence of functions whose bounded variation remains uniformly bounded when $h \rightarrow 0$, then one can extract from $Y^{h}$ a subsequence and find a function of bounded variation $Y$ such that $Y^{h}(x)$ converges to $Y(x)$ at every point $x$.

Given this background we can state our main theorem:

Theorem 6.1. Suppose that the prescribed initial values $U(0), V(0)$ and $W(0)$ at time $t=0$ satisfy

$$
\|U(0)\|_{L^{\infty}}+T V((V, W)(0))<\infty
$$


and satisfy the constraint equations (5.7). Then there exists a solution $U=U(t, x), V=$ $V(t, x), W=W(t, x)$ which is defined for all $x \in \mathbb{R}$ and on a (maximal) time interval $t \in\left[0, T_{\max }\right)$, is a solution of (5.1) (in the sense of distributions) satisfying constraints (5.7) at each time, and has the regularity

$$
\begin{gathered}
\|U(t)\|_{L^{\infty}}+T V((V, W)(t))<\infty, \quad t \in\left[0, T_{\max }\right), \\
\int_{\mathbb{R}}\left|(V, W)(t, x)-(V, W)\left(t^{\prime}, x\right)\right| \mathrm{d} x \leqslant C\left|t-t^{\prime}\right|
\end{gathered}
$$

for some $C>0$ and all $t, t^{\prime} \in\left[0, T_{\max }\right)$. When $T_{\max }<\infty$, then the $L^{\infty}$ norm of the geometry variables $W(t)$ must blow up at $t=T_{\max }$.

In theorem 6.1 the solutions satisfy the equations in the sense of distributions: for instance, for the fluid equations (5.5) this means that

$\iint\left(\theta_{t} V_{1}+\theta_{x} V_{2}\right) \mathrm{d} x \mathrm{~d} t=\iint \theta\left(-V_{1}\left(W_{2}+2 W_{4}\right)-V_{2}\left(2 W_{3}+2 W_{5}\right)-\Sigma W_{2}-2 p W_{4}\right) \mathrm{d} x \mathrm{~d} t$,

and

$\iint\left(\theta_{t} V_{2}+\theta_{x} \Sigma\right) \mathrm{d} x \mathrm{~d} t=-\iint\left(V_{1} W_{3}-V_{2}\left(2 W_{2}+2 W_{4}\right)-\Sigma\left(W_{3}+2 W_{5}\right)+2 p W_{5}\right) \mathrm{d} x \mathrm{~d} t$,

for every smooth function $\theta=\theta(t, x)$ with compact support.

The proof of theorem 6.1 will be based on the Glimm scheme [5]. To establish the existence of a solution to the Cauchy problem we proceed as follows:

- Solve the Riemann problem when the source terms are neglected.

- Design an approximation scheme based on Glimm's random choice method and a suitable fractional step procedure to handle the sources.

- Estimate the $L^{\infty}$ norm of the approximate solutions.

- Derive a bound on the total variation of the variables $V$ and $W$.

- Check the compactness and consistency of the scheme, including the constraint equations.

Observe that the equations (especially (5.2)-(5.4)) are linear in $U$ and an $L^{\infty}$ bound on $U$ is sufficient in the convergence analysis. On the other hand, the equations under consideration are nonlinear in $V$ and $W$ and we must therefore control a stronger norm. The total variation is natural when propagating discontinuities are expected in the solutions.

Note that we are regarding $U, V$ and $W$ as independent variables, and are allowing discontinuities in all of them. Thus constraints (5.7) cannot be imposed throughout the approximation scheme but could be checked afterwards, based on the evolution equations (5.10). Alternatively, one could, at each time step, project the approximate solution onto the manifold determined by the constraints. This is probably useful to improve the accuracy in numerical calculations, although it is not required for the convergence analysis.

Remark 6.2. It can be checked that the solution $U$ in theorem 6.1 is weakly continuous in time, in the sense that for every smooth, compactly supported function $\theta$ there exists $C>0$ such that

$$
\left|\int_{\mathbb{R}}\left(U(t, x)-U\left(t^{\prime}, x\right)\right) \theta(x) \mathrm{d} x\right| \leqslant C\left|t-t^{\prime}\right|
$$

for all $t, t^{\prime} \in\left[0, T_{\max }\right)$. 


\section{The Riemann problem}

Throughout this section we neglect the source terms in (5.1) as well as the constraint equations (5.7). The fluid variables satisfy the two conservation laws

$$
\tau_{t}+S_{x}=0, \quad S_{t}+\Sigma_{x}=0,
$$

which are entirely decoupled from the geometry. The Riemann problem for the special relativistic fluid equations (7.1) corresponding to the piecewise constant, initial data

$$
(\tau, S)(0, x)= \begin{cases}\left(\tau^{l}, S^{l}\right), & x<0, \\ \left(\tau^{r}, S^{r}\right), & x>0,\end{cases}
$$

was solved by Smoller and Temple [18]. It is convenient to introduce the Riemann invariants

$$
r=\frac{1}{2}(w-z) \quad s=\frac{1}{2}(w+z)
$$

where

$$
w=\log \left(\frac{1+v}{1-v}\right)
$$

and

$$
z=\int^{\mu} \frac{\sqrt{p^{\prime}(m)}}{m+p(m)} \mathrm{d} m=\frac{c_{s}}{1+c_{s}^{2}} \log \mu=k_{0} \log \mu .
$$

The mapping $v \in(-1,1) \mapsto w \in \mathbb{R}$ is one-to-one, while the mapping $\mu \mapsto z$ is one-to-one from $(0, \infty)$ onto itself.

The construction of the solution of the Riemann problem (7.1), (7.2) requires some tedious algebra but is rather standard. We will only need here to recall a few properties of the solutions. The solution of the Riemann problem is made of two waves associated with the two nonlinear characteristic families associated with the sound speed $c_{s}$.

(1) The regions

$$
R_{M}=\{(r, s):-M \leqslant r \leqslant s \leqslant M\}, \quad M>0,
$$

are convex in the plane of the conservative variables and are invariant regions for the Riemann solutions, that is, if the Riemann data belong to $R_{M}$ for some $M>0$, then the corresponding Riemann solution also belongs to $R_{M}$. This is established by direct verification from the expressions of the shock curves and rarefaction curves which are used to construct the solution of the Riemann problem.

(2) When two elementary waves of the relativistic fluid system or, more generally, when two Riemann solutions 'interact', the total variation is non-increasing. This is so provided the total variation is measured in the variable $\log \mu$, as first discovered by Nishida for the fluid equation in flat space [13].

We now turn to the equations for the geometric components. Neglecting the source terms we obtain

$$
\begin{aligned}
& U_{1 t}-U_{2 x}+\frac{1}{2} \kappa\left(1+c_{s}^{2}\right)\left(W_{1} \mu\right)_{x}=0 \\
& U_{2 t}-U_{1 x}=0 \\
& U_{3 t}-U_{4 x}-\frac{1}{2} \kappa\left(1-c_{s}^{2}\right)\left(W_{1} \mu\right)_{x}=0, \\
& U_{4 t}-U_{3 x}=0
\end{aligned}
$$

and

$$
U_{5 t}-U_{6 x}=0, \quad U_{6 t}-U_{5 x}=0
$$


Solving the Riemann problem for the linear hyperbolic system (7.6) is straightforward: the solution $\left(U_{5}, U_{6}\right)$ contains two waves with speeds -1 and 1 respectively. Exactly one of the Riemann invariants $U_{5} \pm U_{6}$ is discontinuous at each of the two waves. More precisely, the Riemann problem (7.6) and

$$
\left(U_{5}, U_{6}\right)(0, x)= \begin{cases}\left(U_{5}^{l}, U_{6}^{l}\right), & x<0 \\ \left(U_{5}^{r}, U_{6}^{r}\right), & x>0\end{cases}
$$

are solved with two waves with speeds -1 and +1 . The constant states $\left(U_{5}^{l}, U_{6}^{l}\right)$ and $\left(U_{5}^{r}, U_{6}^{r}\right)$ are separated by a middle constant state

$$
\left(U_{5}^{*}, U_{6}^{*}\right)
$$

determined by the Rankine Hugoniot relations for each wave, that is,

$$
-\left(U_{5}^{*}-U_{6}^{l}\right)-\left(U_{5}^{*}-U_{6}^{l}\right)=0, \quad-\left(U_{6}^{*}-U_{6}^{l}\right)-\left(U_{5}^{*}-U_{5}^{l}\right)=0,
$$

and

$$
\left(U_{5}^{r}-U_{5}^{*}\right)-\left(U_{6}^{r}-U_{6}^{*}\right)=0, \quad\left(U_{6}^{r}-U_{6}^{*}\right)-\left(U_{5}^{r}-U_{5}^{*}\right)=0 .
$$

This is equivalent to the continuity of the first (second) Riemann invariant across the first (second) wave, thus

$$
2 U_{5}^{*}=U_{5}^{l}+U_{6}^{l}+U_{5}^{r}-U_{6}^{r}, \quad 2 U_{6}^{*}=U_{5}^{l}+U_{6}^{l}-U_{5}^{r}+U_{6}^{r} .
$$

This explicit form for the solution will allow us to control easily any norm of the Riemann solution we might need to estimate later in the proof.

Theorem 7.1. Given any constant Riemann data $\left(U^{l}, V^{l}, W^{l}\right)$ and $\left(U^{r}, V^{r}, W^{r}\right)$, there exists a solution to the Riemann problem

$$
\begin{array}{ll}
U_{t}+A^{1} U_{x}+A^{2} V_{x}+A^{3} W_{x}=0, & U=U(t, x) \in \mathbb{R}^{6}, \\
V_{t}+B(V) V_{x}=0, & V=V(t, x) \in \mathbb{R}^{2}, \\
W_{t}+C W_{x}=0, & W=W(t, x) \in \mathbb{R}^{7}, \\
(U, V, W)(0, x)= \begin{cases}\left(U^{l}, V^{l}, W^{l}\right), & x<0, \\
\left(U^{r}, V^{r}, W^{r}\right), & x>0 .\end{cases}
\end{array}
$$

The variable $V$ contains two (shock or rarefaction) waves associated with the sound speeds $\pm c_{s}$, while the geometric variables $U_{5}, U_{6}$ contain two shocks with speeds \pm 1 , and the variables $U_{1}, \ldots, U_{4}$ contain five waves associated with the light speeds \pm 1 , the sound speeds $\pm c_{s}$ and the speed 0. The variable $W_{1}$ has a single wave with zero speed, while $W_{2}, \ldots, W_{7}$ have jumps with speeds \pm 1 .

\section{Remark 7.2.}

- If the components $U_{5}, U_{6}$ are continuous initially that is $U_{5}^{l}=U_{5}^{r}$ and $U_{6}^{l}=U_{6}^{r}$ then $U_{5}, U_{6}$ remain constant. In particular, discontinuities in the components $U_{5}, U_{6}$ cannot appear unless they exist at the initial time.

- The same is true for all of the components of $U_{1}, U_{2}, U_{3}, U_{4}$ along the light cone.

Proof. It remains to solve the Riemann problem for $\left(U_{3}, U_{4}\right)$, for instance. The jump relation along a sound wave with speed $\lambda$ is

$$
\begin{aligned}
& -\lambda\left(U_{3}^{+}-U_{3}^{-}\right)-\left(U_{4}^{+}-U_{4}^{-}\right)-\frac{1}{2} \kappa W_{1}\left(1-c_{s}^{2}\right)\left(\mu^{+}-\mu^{-}\right)=0, \\
& -\lambda\left(U_{4}^{+}-U_{4}^{-}\right)-\left(U_{3}^{+}-U_{3}^{-}\right)=0 .
\end{aligned}
$$


The right-hand components can be expressed in terms of the left-hand components:

$$
\begin{aligned}
& U_{3}^{+}=U_{3}^{-}+\frac{\lambda}{1-\lambda^{2}} \frac{1}{2} \kappa W_{1}\left(1-c_{s}^{2}\right)\left(\mu^{+}-\mu^{-}\right), \\
& U_{4}^{+}=U_{4}^{-}-\frac{1}{1-\lambda^{2}} \frac{1}{2} \kappa W_{1}\left(1-c_{s}^{2}\right)\left(\mu^{+}-\mu^{-}\right) .
\end{aligned}
$$

It will be convenient to set

$$
W_{1}^{-}=W_{1}^{l}, \quad W_{1}^{+}=W_{1}^{r} .
$$

In the special case that the fluid variables contain two shock waves we can combine the above jump relations and determine the solutions $\left(U_{3}, U_{4}\right)$ : it is made of five waves and six constant states

$$
\begin{array}{lrr}
\left(U_{3}^{l}, U_{4}^{l}\right), & \left(U_{3}^{l *}, U_{4}^{l *}\right), & \left(U_{3}^{*-}, U_{4}^{*-}\right), \\
\left(U_{3}^{*+}, U_{4}^{*+}\right), & \left(U_{3}^{* r}, U_{4}^{* r}\right), & \left(U_{3}^{r}, U_{4}^{r}\right),
\end{array}
$$

where $\left(U_{3}^{l}, U_{4}^{l}\right)$ and $\left(U_{3}^{r}, U_{4}^{r}\right)$ are given constants and along the wave with speed -1 we have

$$
U_{3}^{l *}+U_{4}^{l *}=U_{3}^{l}+U_{4}^{l},
$$

while along the sound wave with speed $\lambda^{l}$

$$
\begin{aligned}
U_{3}^{*-}-U_{3}^{* l} & =\frac{\lambda^{l}}{1-\left(\lambda^{l}\right)^{2}} \frac{1}{2} \kappa W_{1}^{-}\left(1-c_{s}^{2}\right)\left(\mu^{*}-\mu^{l}\right), \\
U_{4}^{*-}-U_{4}^{* l} & =-\frac{1}{1-\left(\lambda^{l}\right)^{2}} \frac{1}{2} \kappa W_{1}^{-}\left(1-c_{s}^{2}\right)\left(\mu^{*}-\mu^{l}\right),
\end{aligned}
$$

and for the stationary wave

$$
U_{3}^{*-}-U_{3}^{*+}=0, \quad U_{4}^{*+}-U_{4}^{*-}+\frac{1}{2} \kappa\left(W_{1}^{+}-W_{1}^{-}\right)\left(1-c_{s}^{2}\right) \mu^{*}=0 .
$$

Finally along the sound wave with speed $\lambda^{r}$

$$
\begin{aligned}
& U_{3}^{r *}-U_{3}^{*+}=\frac{\lambda^{r}}{1-\left(\lambda^{r}\right)^{2}} \frac{1}{2} \kappa W_{1}^{+}\left(1-c_{s}^{2}\right)\left(\mu^{r}-\mu^{*}\right), \\
& U_{4}^{r *}-U_{4}^{*+}=-\frac{1}{1-\left(\lambda^{r}\right)^{2}} \frac{1}{2} \kappa W_{1}^{+}\left(1-c_{s}^{2}\right)\left(\mu^{r}-\mu^{*}\right),
\end{aligned}
$$

and for the wave with speed +1

$$
U_{3}^{* r}-U_{4}^{* r}=U_{3}^{r}-U_{4}^{r} \text {. }
$$

Note that all of the constants $\tau^{*}, \Sigma^{*}, \lambda^{l}$ and $\lambda^{r}$ are already known from solving the Riemann problem in the fluid variables. The above linear, algebraic system of eight equations determines uniquely the eight unknowns $U_{3}^{* l}, U_{4}^{* l}, U_{3}^{*-}, U_{4}^{*-}, U_{3}^{*+}, U_{4}^{*+}, U_{3}^{* r}$ and $U_{4}^{* r}$. Setting

$$
\alpha^{l}=\frac{1}{2} \kappa W_{1}^{-} \frac{\left(1-c_{s}^{2}\right)}{1-\left(\lambda^{l}\right)^{2}}\left(\mu^{*}-\mu^{l}\right), \quad \alpha^{r}=\frac{1}{2} \kappa W_{1}^{+} \frac{\left(1-c_{s}^{2}\right)}{1-\left(\lambda^{r}\right)^{2}}\left(\mu^{r}-\mu^{*}\right)
$$

and

$$
\beta^{*}=\frac{1}{2} \kappa\left(W_{1}^{+}-W_{1}^{-}\right)\left(1-c_{s}^{2}\right) \mu^{*},
$$

we find

$$
\begin{aligned}
& U_{3}^{* l}=\frac{1}{2}\left[U_{3}^{l}+U_{3}^{r}+U_{4}^{l}-U_{4}^{r}-\left(1+\lambda^{l}\right) \alpha^{l}-\left(1+\lambda^{r}\right) \alpha^{r}\right] \\
& U_{4}^{* l}=\frac{1}{2}\left[U_{3}^{l}-U_{3}^{r}+U_{4}^{l}+U_{4}^{r}+\left(1+\lambda^{l}\right) \alpha^{l}+\left(1+\lambda^{r}\right) \alpha^{r}+\beta^{*}\right]
\end{aligned}
$$




$$
\begin{aligned}
& U_{3}^{*-}=U_{3}^{*+}=\frac{1}{2}\left[U_{3}^{l}+U_{3}^{r}+U_{4}^{l}-U_{4}^{r}+\left(-1+\lambda^{l}\right) \alpha^{l}-\left(1+\lambda^{r}\right) \alpha^{r}-\beta^{*}\right] \\
& U_{4}^{*-}=\frac{1}{2}\left[U_{3}^{l}-U_{3}^{r}+U_{4}^{l}+U_{4}^{r}+\left(-1+\lambda^{l}\right) \alpha^{l}+\left(1+\lambda^{r}\right) \alpha^{r}+\beta^{*}\right] \\
& U_{4}^{*+}=\frac{1}{2}\left[U_{3}^{l}-U_{3}^{r}+U_{4}^{l}+U_{4}^{r}+\left(-1+\lambda^{l}\right) \alpha^{l}+\left(1+\lambda^{r}\right) \alpha^{r}-\beta^{*}\right] \\
& U_{3}^{* r}=\frac{1}{2}\left[U_{3}^{l}+U_{3}^{r}+U_{4}^{l}-U_{4}^{r}+\left(-1+\lambda^{l}\right) \alpha^{l}+\left(-1+\lambda^{r}\right) \alpha^{r}-\beta^{*}\right] \\
& U_{4}^{* r}=\frac{1}{2}\left[U_{3}^{l}-U_{3}^{r}+U_{4}^{l}+U_{4}^{r}+\left(-1+\lambda^{l}\right) \alpha^{l}+\left(-1+\lambda^{r}\right) \alpha^{r}-\beta^{*}\right]
\end{aligned}
$$

A similar result holds for $U_{1}$ and $U_{2}$. Note that jumps in the geometry across the four interfaces occur if and only if there are jumps in the matter variables across the inner pair of interfaces. Note too that this Riemann solution violates the constraint equations (3.9) and (3.10). Jumps in $U_{3}=b_{t x}$ and $U_{4}=b_{x x}$ can occur only at those interfaces where the matter variables are discontinuous.

This completes the construction of the Riemann solution in the variables $\left(U_{3}, U_{4}\right)$ when there are only shock waves.

If the fluid variables have a rarefaction fan, we can determine $U_{3}, U_{4}$ within the rarefaction:

$$
-\xi U_{3}^{\prime}-U_{4}^{\prime}-\frac{1}{2} \kappa W_{1}\left(1-c_{s}^{2}\right) \mu^{\prime}=0, \quad-\xi U_{4}^{\prime}-U_{3}^{\prime}=0,
$$

where $\xi=x / t$, and $a$ is a constant. Therefore the rarefaction fan (and thus the rarefaction curve $)$ in the variable $\left(U_{3}, U_{4}\right)$ issuing from a left-hand state $\left(U_{3}^{-}, U_{4}^{-}\right)$is given by the explicit formula $\left(\xi \in\left[\lambda^{-}, \lambda^{+}\right]\right)$,

$$
\begin{aligned}
& U_{3}(\xi)=U_{3}^{-}+\int_{\lambda^{-}}^{\xi} \frac{x}{1-x^{2}} \frac{1}{2} \kappa W_{1}\left(1-c_{s}^{2}\right) \mu^{\prime}(x) \mathrm{d} x \\
& U_{4}(\xi)=U_{4}^{-}-\int_{\lambda^{-}}^{\xi} \frac{1}{1-x^{2}} \frac{1}{2} \kappa W_{1}\left(1-c_{s}{ }^{2}\right) \mu^{\prime}(x) \mathrm{d} x .
\end{aligned}
$$

Here $\lambda^{-}, \lambda^{+}$are the smallest and largest speeds of the rarefaction fan and $\mu=\mu(\xi)$ denotes the energy density within the fan. We end up with explicit expressions for the rarefaction. When the left-hand or right-hand sound waves are rarefaction fans, the jump conditions (7.12) and (7.13) should be replaced by

$$
\begin{aligned}
& U_{3}^{*}=U_{3}^{l *}+\int_{\lambda^{-l}}^{\lambda^{+l}} \frac{x}{1-x^{2}} \frac{1}{2} \kappa W_{1}^{-}\left(1-c_{s}^{2}\right) \mu^{l^{\prime}}(x) \mathrm{d} x, \\
& U_{4}^{*}=U_{4}^{l *}-\int_{\lambda^{-l}}^{\lambda^{+l}} \frac{1}{1-x^{2}} \frac{1}{2} \kappa W_{1}^{-}\left(1-c_{s}^{2}\right) \mu^{l^{\prime}}(x) \mathrm{d} x,
\end{aligned}
$$

and

$$
\begin{aligned}
U_{3}^{* r} & =U_{3}^{*}+\int_{\lambda^{-r}}^{\lambda^{+r}} \frac{x}{1-x^{2}} \frac{1}{2} \kappa W_{1}^{+}\left(1-c_{s}^{2}\right) \mu^{r^{\prime}}(x) \mathrm{d} x, \\
U_{4}^{* r} & =U_{4}^{*}-\int_{\lambda^{-r}}^{\lambda^{+r}} \frac{1}{1-x^{2}} \frac{1}{2} \kappa W_{1}^{+}\left(1-c_{s}^{2}\right) \mu^{r \prime}(x) \mathrm{d} x,
\end{aligned}
$$

respectively. This completes the discussion of the Riemann solver.

Remark 7.3. The discussion above is not applicable to the limiting case $c_{s}=1$ because we assumed right at the start that $v \in(-1,1)$. However the limiting case is very simple. We do not use the equation for the $V$-variable, but adjoin $U_{7}=\phi_{x t}$ and $U_{8}=\phi_{x x}$ to the $U$-variable and $W_{8}=\phi_{t}$ and $W_{9}=\phi_{x}$ to the $W$-variable. We need also to simplify the first (5.2) and the second (5.6). Now $U_{7}$ and $U_{8}$ satisfy equations whose principal part is identical to that of (5.4) on replacing $U_{5}$ by $U_{7}$ and $U_{6}$ by $U_{8}$. Then remark 6.2 applies to $U_{7}, U_{8}$. Discontinuities in 
these components cannot appear unless they exist at the initial time. Thus the 'fluid' variables remain continuous. It follows that there can be no discontinuities in the $U$-variable unless they were present initially.

\section{A generalized Glimm scheme}

In this section, we introduce a generalization of the Glimm scheme $[5,10,11]$ to solve the initial value problem and we establish its convergence towards a solution of the system of equations under consideration, under the assumption that no blow up occurs in the geometry terms, that is on any interval $[0, T]$ satisfying

$$
\sup _{t \in[0, T], x \in \mathbb{R}}|W(t, x)|<\infty \text {. }
$$

It will be convenient to set $u=(U, V, W)$ and to rewrite the equations in the form

$$
\partial_{t} u+\partial_{x} f(u)=g(u), \quad u(t, x) \in \Omega \subset \mathbb{R}^{15}, \quad t>0, x \in \mathbb{R},
$$

The precise expressions of $f, g, \Omega$ are easily deduced from (5.1). The approximation scheme can be decomposed into a step based on a (generalized) Riemann solver, and a step based on an ODE solver.

We start by analysing the properties of the source term $g(u)$. For every $u_{0} \in \Omega$ we denote by $u(t)=\mathbf{S}_{t}\left(u_{0}\right)$ the solution of the ordinary differential equation

$$
u^{\prime}(t)=g(u(t)), \quad t \geqslant 0, \quad u(0)=u_{0} .
$$

In general, the solution of (8.2) is defined on a finite time interval only, since $u(t)$ may tend to infinity in finite time. We shall also use the notation $\mathbf{S}_{t}\left(u_{0}\right)=(U, V, W)(t), u_{0}=$ $\left(U_{0}, V_{0}, W_{0}\right)$, etc.

Lemma 8.1 (Uniform $L^{\infty}$ bounds). For every $u_{0} \in \Omega$ the solution $\mathbf{S}_{t}\left(u_{0}\right)$ of the problem (8.2) satisfies the following property: as long as the geometry variables $W$ remain bounded, the geometry variables $U$ and the fluid variables $V$ remain also bounded and, furthermore, the fluid variables remain bounded away from the singularities $\mu=0$ and $v= \pm 1$. The Riemann invariant variables of the fluid (defined in section 7) satisfy the estimate

$$
|w(t)|+|z(t)| \leqslant\left|w_{0}\right|+\left|z_{0}\right|+C_{*} t, \quad t \leqslant T,
$$

where $C_{*}$ is a constant depending only on $\sup _{t \in[0, T]}|W(t)|$ and $T$ is any time such that this supremum is finite.

Proof. We first show that, as long as $U, W$ remain bounded, we have

$$
0 \leqslant \mu \leqslant C, \quad-1<v<1 .
$$

To this end we rewrite the $V$-system in terms of the variables $\mu, v$. Neglecting the space derivatives in the fluid equations we find

$$
\tau_{t}=T_{1}(V, W), \quad S_{t}=T_{2}(V, W),
$$

where $T_{1}$ and $T_{2}$ were defined in (3.19). It is straightforward to replace $\tau$ and $S$ by $\mu$ and $v$, finding

$$
\mu_{t}=f(\mu, v), \quad v_{t}=g(v),
$$

with

$$
\left\{\begin{array}{l}
f(\mu, v)=-\mu\left(1+c_{s}{ }^{2}\right)\left(\left(1-v^{2}\right) W_{2}+2\left(W_{4}+v W_{5}\right)\right) /\left(1-c_{s}{ }^{2} v^{2}\right) \\
g(v)=\left(1-v^{2}\right)\left(-v\left(1-c_{s}{ }^{2}\right) W_{2}-\left(1-c_{s}{ }^{2} v^{2}\right) W_{3}+2 v c_{s}{ }^{2}\left(W_{4}+v W_{5}\right)\right) /\left(1-c_{s}{ }^{2} v^{2}\right) .
\end{array}\right.
$$


As long as $W_{2}, W_{3}, W_{4}$ and $W_{5}$ remain bounded, $g(v)$ is a smooth function of $v \in(-1,1)$ and $g( \pm 1)=0$. We deduce that trajectories $t \mapsto v(t)$ cannot exit the interval $(-1,1)$ and therefore $|v|<1$.

Note further that $f(\mu, v)$ is linear in $\mu$,

$$
f(\mu, v)=\tilde{f}(v) \mu,
$$

where $\tilde{f}(v)$ is smooth for $|v|<1$ and smooth $a_{t}, b_{t}$ and $b_{x}$. It follows that $\mu$ stays positive and cannot blow up in finite time, unless the geometry variables themselves blow up.

To bound $V$ uniformly we now consider the evolution of the Riemann invariant variables:

$w_{t}=\frac{2}{1-c_{s}{ }^{2} v^{2}}\left(-v\left(1-c_{s}^{2}\right) W_{2}-\left(1-c_{s}{ }^{2} v^{2}\right) W_{3}+2 v c_{s}^{2}\left(W_{4}+v W_{5}\right)\right)$

and

$$
z_{t}=-K_{0} \frac{1+c_{s}^{2}}{1-c_{s}^{2} v^{2}}\left(\left(1-v^{2}\right) W_{2}+2\left(W_{4}+v W_{5}\right)\right)
$$

We see that

$$
w_{t}=B^{w} W, \quad z_{t}=B^{z} W,
$$

where $B^{w}, B^{z}$ are matrices that remain uniformly bounded, since, as we have just established, the velocity variable $v$ stays in the interval $(-1,1)$. As long as the geometry variable $W$ remains bounded, we can conclude that the fluid variables $w, z$ are also bounded. Finally we observe that the equations for $U$, for instance for $U_{1}$ and $U_{2}$,

$$
U_{1 t}=2\left(U_{3} W_{4}-U_{4} W_{5}-U_{5} W_{6}+U_{6} W_{7}\right), \quad U_{2 t}=0,
$$

are linear in $U$ and therefore can be solved as long as $W$ remains bounded. This completes the proof of lemma 8.1.

We now turn to the Riemann problem. Given two constant vectors $u_{L}, u_{R} \in \Omega$ and a point $\left(t_{0}, x_{0}\right)$ the generalized Riemann problem $R_{G}\left(u_{L}, u_{R} ; t_{0}, x_{0}\right)$ is the Cauchy problem for the system (8.1) with piecewise constant initial data

$$
u\left(t_{0}, x\right)= \begin{cases}u_{L}, & x<x_{0}, \\ u_{R}, & x>x_{0} .\end{cases}
$$

On the other hand, the classical Riemann problem $R_{C}\left(u_{L}, u_{R} ; t_{0}, x_{0}\right)$ is obtained by neglecting the source term $g(u)$. Let us denote its solution by $u^{C}\left(t, x ; u_{L}, u_{R} ; t_{0}, x_{0}\right)$. Recall that the solution of the generalized Riemann problem is piecewise smooth and has a local structure similar to the one of the classical Riemann problem. This motivates us to define an approximate solution of it, denoted by $u^{G}\left(t, x ; u_{L}, u_{R} ; t_{0}, x_{0}\right)$ and defined for all $t>t_{0}$ and $x \in \mathbb{R}$, by

$u^{G}\left(t, x ; u_{L}, u_{R} ; t_{0}, x_{0}\right)=u^{C}\left(t, x ; u_{L}, u_{R} ; t_{0}, x_{0}\right)+\int_{0}^{t-t_{0}} g\left(\mathbf{S}_{\tau} u^{C}\left(t, x ; u_{L}, u_{R} ; t_{0}, x_{0}\right)\right) \mathrm{d} \tau$.

Observe that $u^{G}$ at a given time $t$ only depends upon $u^{C}$ at the same time $t$.

We claim that the function $u^{G}$ approximates (in an averaged sense) the system of equations (8.1). For any $s, r>0$ and any smooth function $\theta: \mathbb{R}^{+} \times \mathbb{R} \rightarrow \mathbb{R}$ with compact support, we set

$$
\Delta(s, r ; \theta)=\int_{t_{0}}^{t_{0}+s} \int_{x_{0}-r}^{x_{0}+r}\left(u^{G} \partial_{t} \theta+f\left(u^{G}\right) \partial_{x} \theta+g\left(u^{G}\right) \theta\right) \mathrm{d} x \mathrm{~d} t .
$$

It is not difficult to check the following estimate. 
Lemma 8.2 (Approximate balance law for the Riemann solver). For any $t_{0} \geqslant 0, x_{0} \in$ $\mathbb{R}, u_{L}, u_{R} \in \Omega$, any $s, r>0$, and any smooth, compactly supported function $\theta: \mathbb{R}^{+} \times \mathbb{R} \rightarrow \mathbb{R}$, the function $u^{G}(t, x)=u^{G}\left(t, x ; u_{L}, u_{R} ; t_{0}, x_{0}\right)$ satisfies the estimate

$$
\begin{aligned}
\Delta(s, r ; \theta)- & \int_{x_{0}-r}^{x_{0}+r} u^{G}\left(t_{0}+s, x\right) \theta\left(t_{0}+s, x\right) \mathrm{d} x+\int_{x_{0}-r}^{x_{0}+r} u^{G}\left(t_{0}, x\right) \theta\left(t_{0}, x\right) \mathrm{d} x \\
& \quad-\int_{t_{0}}^{t_{0}+s} f\left(u^{G}\left(t, x_{0}+r\right)\right) \theta\left(t, x_{0}+r\right) \mathrm{d} t+\int_{t_{0}}^{t_{0}+s} f\left(u^{G}\left(t, x_{0}-r\right)\right) \theta\left(t, x_{0}-r\right) \mathrm{d} t \\
= & O(1)\left(s^{2}+r^{2}\right)\left(s+r+\left|u_{R}-u_{L}\right|\right),
\end{aligned}
$$

where $O(1)$ is a bounded function depending on $\theta$ and remaining uniformly bounded when $r, s \rightarrow 0$.

Our generalization of the Glimm method is based on the approximate Riemann solver just introduced. Let $s$ and $r$ denote time and space mesh lengths satisfying $s / r<1$, the ratio $s / r$ being kept constant while $r, s \rightarrow 0$. Let $a=\left(a_{k}\right)_{k=0,1, \ldots}$ be an equidistributed sequence in $(-1,1)$.

We define an approximate solution $u_{r}=u_{r}(t, x)$ of the general Cauchy problem consisting of system (8.1) and prescribed initial data $u_{0}$ :

$$
u(0, x)=u_{0}(x), \quad x \in \mathbb{R} .
$$

First, $u_{r}(0, x)$ is defined to be a piecewise constant approximation of $u_{0}$, say

$$
u_{r}(0, x)=u_{0}((h+1) r), \quad x \in[h r,(h+2) r), \quad h \text { even. }
$$

If $u_{r}(t, x)$ is known for $t<k s$ for some integer $k \geqslant 0$, we set

$u_{r}(k s+, x)=u_{r}\left(k s-,\left(h+1+a_{k}\right) r\right) \quad x \in[h r,(h+2) r), \quad k+h$ even.

Then, in each region $k s \leqslant t<(k+1) s,(h-1) r \leqslant x<(h+1) r(k+h$ even $)$, the function $u_{r}$ is defined as the approximate solution to the generalized Riemann problem

$$
R_{G}\left(u_{r}(k s,(h-1) r), u_{r}(k s,(h+1) r) ; k s, h r\right),
$$

that is (by our definition of the approximate solver)

$$
\begin{aligned}
& u_{r}(t, x)=u^{G}\left(t, x ; u_{r}(k s,(h-1) r), u_{r}(k s,(h+1) r) ; k s, h r\right) \\
& t \in[k s,(k+1) s), x \in[(h-1) r,(h+1) r), \quad k+h \text { even. }
\end{aligned}
$$

This completes the description of the scheme.

We will use the notation

$$
u_{r}=\left(U_{r}, V_{r}, W_{r}\right) .
$$

Based on lemma 8.1 we have :

Lemma 8.3 ( $L^{\infty}$ Stability of the generalized Glimm scheme). As long as the geometry variables $W_{r}$ remain uniformly bounded, then the fluid variables $V_{r}$ remain uniformly bounded and, furthermore, remain bounded away from the singularities $\mu=0$ and $v= \pm 1$. The density $\mu_{r}$ and velocity $v_{r}$ associated with $V_{r}$ satisfy, for every $T$ before blow up, the estimates

$c_{*} \leqslant \mu_{r}(t, x) \leqslant C_{*}, \quad\left|v_{r}(t, x)\right| \leqslant 1-c_{*}, \quad t \in[0, T), x \in \mathbb{R}$,

where $c_{*} \in(0,1)$ and $C_{*}>0$ depend only on $T$ and

$$
\sup _{t \in[0, T], x \in \mathbb{R}}\left|W_{r}(t, x)\right| \text {. }
$$


Proof. The lemma follows directly from the following two observations:

(1) In view of lemma 6.1, the ODE solver ensures constancy of the sign of the density variable and keeps the velocity variable within the physically admissible interval $(-1,1)$. Moreover, the $L^{\infty}$ norm of $\mu_{r}$ and $v_{r}$ can increase after each ODE step but, for each time step, the increase is at most $C_{*} S$ (see (8.3)).

(2) On the other hand, the Riemann solver satisfies a maximum principle in the Riemann invariants associated with the fluid variables (see Smoller and Temple [18]).

Our scheme combines the two effects above. Adding up the various contributions $C_{*}$ we deduce that, over the time interval $[0, T]$,

$$
\sup _{x}\left|w_{r}(t, x)\right|+\sup _{x}\left|z_{r}(t, x)\right| \leqslant \sup _{x}\left|w_{0}(x)\right|+\sup _{x}\left|z_{0}(x)\right|+C_{*} T, \quad t \leqslant T .
$$

Rewriting this estimate in terms of the density and velocity gives precisely (8.15). This completes the proof of lemma 8.3.

We now establish the uniform stability in total variation for $V$ and $W$, together with the $L^{\infty}$ stability of $U_{r}$. To simplify the notation we set

$$
u_{k, h+1}=u_{r}(k s+,(h+1) r), \quad k+h \text { even, }
$$

which, by construction, has the form

$$
u_{k, h+1}=\tilde{u}_{k, h+1}+\int_{0}^{s} g\left(\mathbf{S}_{\tau} \tilde{u}_{k, h+1}\right) \mathrm{d} \tau, \quad k+h \text { even }
$$

where $\widetilde{u}_{k, h}$ is a value taken by the solution of the classical Riemann problem

$$
R_{C}\left(u_{k-1, h}, u_{k-1, h+2} ;(k-1) s,(h+1) r\right) .
$$

Using earlier notation we actually have

$$
\tilde{u}_{k, h+1}=u^{C}\left(a_{k} \frac{r}{s} ; u_{k-1, h}, u_{k-1, h+2}\right) .
$$

Following Glimm [5] we decompose the $(t, x)$-plane into diamonds $\Delta_{k, h}$ (here $k+h$ is even) with centre $(k s, h r)$ and vertices

$$
\begin{array}{ll}
S=\left((k-1) s,\left(h+a_{k-1}\right) r\right), & W=\left(k s,\left(h-1+a_{k}\right) r\right), \\
E=\left(k s,\left(h+1+a_{k}\right) r\right), & N=\left((k+1) s,\left(h+a_{k+1}\right) r\right) .
\end{array}
$$

For a fixed pair $(k, h)$ we set

$$
u_{S}=u_{k-1, h}, \quad u_{W}=u_{k, h-1}, \quad u_{E}=u_{k, h+1}, \quad u_{N}=u_{k+1, h},
$$

and

$$
\tilde{u}_{W}=\tilde{u}_{k, h-1}, \quad \tilde{u}_{E}=\tilde{u}_{k, h+1}, \quad \tilde{u}_{N}=\tilde{u}_{k+1, h} .
$$

By construction, $u_{W}$ and $u_{E}$ are values achieved by approximate Riemann solutions at the points $((k-1) s,(h-1) r)$ and $((k-1) s,(h+1) r)$ respectively, more precisely

$$
u_{W}=\tilde{u}_{W}+\int_{0}^{s} g\left(\mathbf{S}_{\tau} \widetilde{u}_{W}\right) \mathrm{d} \tau
$$

and

$$
u_{E}=\tilde{u}_{E}+\int_{0}^{s} g\left(\mathbf{S}_{\tau} \tilde{u}_{E}\right) \mathrm{d} \tau
$$

Similarly, we have

$$
u_{N}=\tilde{u}_{N}+\int_{0}^{s} g\left(\mathbf{S}_{\tau} \tilde{u}_{N}\right) \mathrm{d} \tau .
$$


We define the strength vector $\epsilon\left(u_{L}, u_{R}\right) \in \mathbb{R}^{9}$ from the jumps of the solutions of the Riemann problem with data $u_{L}, u_{R}$ : the first two components $\epsilon_{1}\left(u_{L}, u_{R}\right)$ and $\epsilon_{2}\left(u_{L}, u_{R}\right)$ are the jumps of the log of the density across the fluid shock or rarefaction waves; the remaining six components of $\epsilon\left(u_{L}, u_{R}\right)$ are associated with the geometric variable $W$ and are the jumps in the corresponding Riemann invariants (along the waves with light speed \pm 1 ). It is important to note that $\epsilon$ depends only on the $V, W$ variables. This is consistent with the fact that we do not need to control the total variation in $U$. The norm $\left|\epsilon\left(u_{L}, u_{R}\right)\right|$ is defined to be the sum of the absolute values of the components. All the estimates below involve constants that depend on the geometry for which we assume uniform boundedness.

Lemma 8.4. The wave strength $\epsilon: \Omega \times \Omega \rightarrow \mathbb{R}^{9}$ is a smooth function of its arguments. For any $u_{L}, u_{R}, u_{L}^{\prime}, u_{R}^{\prime}$ (in any given compact subset of $\Omega$ ) we have the uniform estimate

$$
\begin{aligned}
\epsilon\left(u_{L}^{\prime}, u_{R}^{\prime}\right)=\epsilon & \left(u_{L}, u_{R}\right)+O(1)\left|\epsilon\left(u_{L}, u_{R}\right)\right|\left(\left|u_{L}^{\prime}-u_{L}\right|+\left|u_{R}^{\prime}-u_{R}\right|\right) \\
& +O(1)\left|\left(u_{R}^{\prime}-u_{L}^{\prime}\right)-\left(u_{R}-u_{L}\right)\right| .
\end{aligned}
$$

Proof. Note that $\epsilon_{i}\left(u_{L}, u_{R}\right)=0$ if $u_{R}=u_{L}$, so that

$$
\epsilon_{i}\left(u_{L}, u_{R}\right)=\int_{0}^{1} \frac{\partial \epsilon_{i}}{\partial u_{r}}\left(u_{L},(1-\tau) u_{L}+\tau u_{R}\right)\left(u_{R}-u_{L}\right) \mathrm{d} \tau
$$

and similarly

$$
\epsilon_{i}\left(u_{L}^{\prime}, u_{R}^{\prime}\right)=\int_{0}^{1} \frac{\partial \epsilon_{i}}{\partial u_{r}}\left(u_{L}^{\prime},(1-\tau) u_{L}^{\prime}+\tau u_{R}^{\prime}\right)\left(u_{R}^{\prime}-u_{L}^{\prime}\right) \mathrm{d} \tau .
$$

We thus get

$$
\begin{aligned}
\epsilon_{i}\left(u_{L}^{\prime}, u_{R}^{\prime}\right)- & \epsilon_{i}\left(u_{L}, u_{R}\right) \\
= & \int_{0}^{1}\left[\frac{\partial \epsilon_{i}}{\partial u_{R}}\left(u_{L}^{\prime},(1-\tau) u_{L}^{\prime}+\tau u_{R}^{\prime}\right)-\frac{\partial \epsilon_{i}}{\partial u_{R}}\left(u_{L},(1-\tau) u_{L}+\tau u_{R}\right)\right]\left(u_{R}-u_{L}\right) \mathrm{d} \tau \\
& +\int_{0}^{1} \frac{\partial \epsilon_{i}}{\partial u_{R}}\left(u_{L}^{\prime},(1-\tau) u_{L}^{\prime}+\tau u_{R}^{\prime}\right)\left(\left(u_{R}^{\prime}-u_{L}^{\prime}\right)-\left(u_{R}-u_{L}\right)\right) \mathrm{d} \tau \\
= & O(1)\left(\left|u_{L}^{\prime}-u_{L}^{\prime}\right|+\left|u_{R}^{\prime}-u_{R}^{\prime}\right|\right)\left|u_{R}-u_{L}\right|+O(1)\left|\left(u_{R}^{\prime}-u_{L}^{\prime}\right)-\left(u_{R}-u_{L}\right)\right|,
\end{aligned}
$$

which leads to the desired estimate since

$$
\left|u_{R}-u_{L}\right|=O(1)\left|\epsilon\left(u_{L}, u_{R}\right)\right| \text {. }
$$

Relying on notation (8.16) we define the strength of the waves entering the diamond $\Delta_{k, h}(k+h$ even $)$ as

$$
\epsilon_{*}\left(\Delta_{k, h}\right)=\left|\epsilon\left(\tilde{u}_{W}, u_{S}\right)\right|+\left|\epsilon\left(u_{S}, \tilde{u}_{E}\right)\right|,
$$

and the strength of the waves leaving the diamond $\Delta_{k, h}$ as

$$
\epsilon^{*}\left(\Delta_{k, h}\right)=\left|\epsilon\left(u_{W}, \tilde{u}_{N}\right)\right|+\left|\epsilon\left(\tilde{u}_{N}, u_{E}\right)\right| .
$$

We consider spacelike curves $J$ connecting vertices of the form $\left(k s,\left(h+1+a_{k}\right) r\right)(k+h$ even). If $J$ contains the segment from $\left((k-1) s,\left(h+a_{k-1}\right) r\right)$ to $\left(k s,\left(h+1+a_{k}\right) r\right)$, one says that the waves $\left(u_{k-1, h}, \tilde{u}_{k, h+1}\right)$ cross $J$. We use the same terminology for the waves $\left(\widetilde{u}_{k, h-1}, u_{k-1, h}\right)$. The total variation along $J$ is then defined by

$$
L(J)=\sum_{\substack{\text { waves } \\ \text { crossing } J}}\left|\epsilon\left(u_{k-1, h}, \widetilde{u}_{k, h+1}\right)\right|+\left|\epsilon\left(\widetilde{u}_{k, h-1}, u_{k-1, h}\right)\right|,
$$


which can also be decomposed according to its components $V, W$ :

$$
L(J)=L_{V}(J)+L_{W}(J) .
$$

We will also control the sup norm of the variable $U$ :

$$
S_{U}(J)=\sup _{J} N\left(U_{r}\right)
$$

where, for every function $U=U(x), N(U)$ is defined from the Riemann invariants $U_{1} \pm U_{2}, U_{3} \pm U_{4}$ and $U_{5} \pm U_{6}$ as

$$
N(U)=\sup _{\mathbb{R}}\left|U_{1} \pm U_{2}\right|+\left|U_{3} \pm U_{4}\right|+\left|U_{5} \pm U_{6}\right|
$$

A spacelike curve $J_{2}$ is said to be an immediate successor of a spacelike curve $J_{1}$ if the curves $J_{1}$ and $J_{2}$ pass through the same vertices, except for one, and if $J_{2}$ lies to the future of the curve $J_{1}$.

Lemma 8.5. Let $J_{1}$ and $J_{2}$ be two I-curves, $J_{2}$ being an immediate successor of $J_{1}$. Denote by $\Delta_{k, h}$ the diamond limited by these curves. Then we have

$L_{V}\left(J_{2}\right)-L_{V}\left(J_{1}\right) \leqslant C_{*} s \epsilon_{*}\left(\Delta_{k, h}\right), \quad L_{W}\left(J_{2}\right)-L_{W}\left(J_{1}\right) \leqslant C_{*} s \epsilon_{*}\left(\Delta_{k, h}\right)$,

where $C$ only depends on the amplitude of the geometry variables $W_{r}$. Furthermore, the $L^{\infty}$ norm in the $U$-variable is controlled by

$$
S_{U}(J) \leqslant\left(1+C_{*} T\right) \sup _{K} L(K),
$$

where the supremum is taken over all spacelike curves.

Proof. Let $u_{S}, u_{W}, u_{E}, u_{N}$ be the values taken by the function $u_{r}$ at the vertices of $\Delta_{k, h}$. We find

$$
\begin{aligned}
L\left(J_{2}\right)-L\left(J_{1}\right) & =\left|\epsilon\left(u_{W}, \widetilde{u}_{N}\right)\right|+\left|\epsilon\left(\widetilde{u}_{N}, u_{E}\right)\right|-\left|\epsilon\left(\widetilde{u}_{W}, u_{S}\right)\right|-\left|\epsilon\left(u_{S}, \widetilde{u}_{E}\right)\right|, \\
& =\epsilon^{*}\left(\Delta_{k, h}\right)-\epsilon_{*}\left(\Delta_{k, h}\right),
\end{aligned}
$$

by definition of $\epsilon_{*}$ and $\epsilon^{*}$.

Assume first that the effect of the source term $g(u)$ can be neglected. Then, the total variation in $V$ (measured with $\log \mu$ ) is non-increasing [18],

$$
L_{V}\left(J_{2}\right)-L_{V}\left(J_{1}\right) \leqslant 0
$$

while the total variation in $W$ (the left-hand side of (5.6) is linear) is conserved,

$$
L_{W}\left(J_{2}\right)-L_{W}\left(J_{1}\right)=0 .
$$

This completes the derivation of (8.18) when the source term is neglected.

We now include the effect of the source term $g(u)$ and consider the decomposition

$$
\begin{aligned}
& L\left(J_{2}\right)-L\left(J_{1}\right)=X_{1}+X_{2}, \\
& X_{1}=\left|\epsilon\left(\widetilde{u}_{W}, \widetilde{\widetilde{u}}_{N}\right)\right|+\left|\epsilon\left(\widetilde{\widetilde{u}}_{N}, \widetilde{u}_{E}\right)\right|-\left|\epsilon\left(\widetilde{u}_{W}, u_{S}\right)\right|-\left|\epsilon\left(u_{S}, \widetilde{u}_{E}\right)\right|, \\
& X_{2}=\left|\epsilon\left(u_{W}, \tilde{u}_{N}\right)\right|+\left|\epsilon\left(\widetilde{u}_{N}, u_{E}\right)\right|-\left|\epsilon\left(\widetilde{u}_{W}, \widetilde{\tilde{u}}_{N}\right)\right|+\left|\epsilon\left(\widetilde{\tilde{u}}_{N}, \widetilde{u}_{E}\right)\right|,
\end{aligned}
$$

where $\widetilde{u}_{N}$ is obtained from $\widetilde{u}_{N}$ by applying the ODE solver backwards. Now, we observe that the discussion in the first part of this proof applies to the term $X_{1}$, while for the term $X_{2}$ we can rely on lemma 8.2 and obtain

$$
\begin{aligned}
\left|X_{2}\right| & \leqslant\left|\epsilon\left(u_{W}, \tilde{u}_{N}\right)\right| C s+\left|\epsilon\left(\tilde{u}_{N}, u_{E}\right)\right| C s+C\left|u_{W}-\tilde{u}_{N}-\tilde{u}_{W}+\widetilde{u}_{N}\right|+C\left|u_{E}-\tilde{u}_{N}-\tilde{u}_{E}+\widetilde{\tilde{u}}_{N}\right| \\
& \leqslant C s\left(\left|\epsilon\left(u_{W}, \tilde{u}_{N}\right)\right|+\left|\epsilon\left(\tilde{u}_{N}, u_{E}\right)\right|\right) .
\end{aligned}
$$


Hence, the total variation of the geometric variable $W$ satisfies

$$
L_{W}\left(J_{2}\right)-L_{W}\left(J_{1}\right) \leqslant C s \epsilon_{*}\left(\Delta_{k, h}\right) .
$$

The change of the total variation of the geometric variables, that is $L_{W}\left(J_{2}\right)-L_{W}\left(J_{1}\right)$, is controlled by the total variation of the right-hand side.

To estimate the $L^{\infty}$ norm of $U$ we rely on the proof of theorem 6.1 in section 6 , which enabled us to derive explicit formula for the Riemann solution. It shows that, when the source term is neglected, the $L^{\infty}$ norm in $U$ increases proportionally to the total variation in $\mu$ and $W_{1}$. The Riemann invariant $U_{1}-U_{2}$ for instance 'propagates' with the light speed and, therefore, waves associated with the sound speed contribute at most once to the $L^{\infty}$ norm in $U$. In other words, we have

$$
S_{U}(J) \leqslant \sup _{K} L(K),
$$

by taking the supremum over all spacelike curves originating on the initial axis $t=0$ and ending on the curve $J$. When the source term is taken into account we follow the same discussion above for the variables $V$ and $W$ and find

$$
S_{U}(J) \leqslant \sup _{K} L(K)+C_{*} T \sup _{K} L(K) .
$$

This completes the proof of lemma 8.5.

For each $k_{0}$ denote by $J_{k_{0}}$ the spacelike curve connecting the points of the form $\left(k s,\left(h+a_{k}\right) r\right)$ with $k=k_{0}$ or $k=k_{0}+1$. By induction from lemma 8.5 we have immediately

$$
\begin{aligned}
L\left(J_{k_{0}+1}\right) & \leqslant L\left(J_{k_{0}}\right)+C_{*} s \sum_{h} \epsilon_{*}\left(\Delta_{k_{0}, h}\right) \\
& =\left(1+C_{*} s\right) L\left(J_{k_{0}}\right) \\
& \leqslant \mathrm{e}^{C_{*} T} L\left(J_{1}\right),
\end{aligned}
$$

where $L\left(J_{1}\right) \leqslant C T V\left(V_{0}, W_{0}\right)$.

We have now established the following result.

Theorem 8.6. Let the initial data $u_{0}: \mathbb{R} \rightarrow \Omega$ be a function with bounded variation. Consider the approximate solutions $u_{r}=\left(U_{r}, V_{r}, W_{r}\right)$ constructed by the generalized version of the Glimm scheme, as defined above. Suppose that on some interval $[0, T]$ the geometry variables remain uniformly bounded, that is

$$
\sup _{t \in[0, T], x \in \mathbb{R}}\left|W_{r}(t, x)\right| \leqslant C_{*}^{\prime}
$$

for some $C_{*}^{\prime}$ independent of $r$.

Then, there exist constants $c_{*} \in(0,1)$ and $C_{*}>0$ depending on $C_{*}^{\prime}$ and $T$, such that the approximate solutions $u_{r}:[0, T] \times \mathbb{R} \rightarrow \Omega$ remain uniformly bounded in $L^{\infty}$ and for $t \in[0, T]$

$$
\begin{aligned}
& c_{*} \leqslant \mu_{r}(t, x) \leqslant C_{*}, \quad\left|v_{r}(t, x)\right| \leqslant 1-c_{*}, \\
& \left\|U_{r}(t)\right\|_{L^{\infty}(\mathbb{R})} \leqslant\left\|U_{r}\right\|_{L^{\infty}(\mathbb{R})}+C_{*} T V\left(\left(V_{0}, W_{0}\right)\right), \\
& T V\left(\left(V_{r}, W_{r}\right)(t)\right) \leqslant C_{*} T V\left(\left(V_{0}, W_{0}\right)\right),
\end{aligned}
$$

and are Lipschitz continuous in time, i.e. for every $0 \leqslant t \leqslant t^{\prime}$

$\int_{\mathbb{R}}\left|\left(V_{r}, W_{r}\right)(t, x)-\left(V_{r}, W_{r}\right)\left(t^{\prime}, x\right)\right| \mathrm{d} x \leqslant\left(\left|t-t^{\prime}\right|+s\right) C_{*} T V\left(\left(V_{0}, W_{0}\right)\right)$. 
We conclude this section with:

Theorem 8.7 (Convergence of the generalized Glimm scheme). Under the assumptions of theorem 8.6 and for almost all equidistributed sequences $a=\left(a_{k}\right)$ there exists a subsequence of $u_{r}$ such that $\left(V_{r}, W_{r}\right)$ converges strongly in the $L^{1}$ norm to a limit $(V, W)$ and $U_{r}$ converges in the averaged sense (weak-star sense in $\left.L^{\infty}\right)$ to some limit $U$. The function $(U, V, W)$ is a weak solution (in the integral sense) to the Cauchy problem (8.1) and (8.6), satisfying moreover

$$
\begin{aligned}
& c_{*} \leqslant \mu(t, x) \leqslant C_{*}, \quad|v(t, x)| \leqslant 1-c_{*}, \\
& T V((V, W)(t)) \leqslant C_{*} T V\left(\left(V_{0}, W_{0}\right)\right),
\end{aligned}
$$

and for every $t, t^{\prime} \leqslant 0$

$$
\int_{\mathbb{R}}\left|(V, W)(t, x)-(V, W)\left(t^{\prime}, x\right)\right| \mathrm{d} x \leqslant\left|t-t^{\prime}\right| C_{*} T V\left(\left(V_{0}, W_{0}\right)\right) .
$$

Our main result, theorem 6.1, follows now from theorems 8.6 and 8.7.

Proof. In view of the uniform estimate in theorem 8.6 and by Helly's compactness theorem it is standard matter to extract a subsequence of approximate solutions (still denoted $u_{r}$ ) such that $\left(V_{r}, W_{r}\right)$ converges strongly in $L^{1}$ to some limit $(V, W): \mathbb{R}^{+} \times \mathbb{R} \rightarrow \mathbb{R}^{9}$ while $U_{r}$ converges weakly to some limit $U$. All the estimates known for $u_{r}$ extend to the limit function $u=(U, V, W)$.

Relying on lemma 8.2 we now show that $u$ is a weak solution of (8.1). Let $\theta: \mathbb{R}^{+} \times \mathbb{R} \rightarrow \mathbb{R}$ be a smooth function with compact support and set

$$
\Delta\left(u_{r}, \theta\right)=\iint_{\mathbb{R}^{+} \times \mathbb{R}}\left(u_{r} \partial_{t} \theta+f\left(u_{r}\right) \partial_{x} \theta+g\left(u_{r}\right) \theta\right) \mathrm{d} x \mathrm{~d} t .
$$

Using that $U_{r}$ converges weakly and $V_{r}, W_{r}$ converge strongly, while all the equations under consideration are linear in $U$ (but nonlinear in $V, W$ ), it follows that, by Lebesgue dominated convergence theorem, as $r \rightarrow 0$

$$
\Delta\left(u_{r}, \theta\right) \rightarrow \Delta(u, \theta) .
$$

We are going to prove that $\Delta\left(u_{r}, \theta\right)$ tends to zero, which will establish that $\Delta(u, \theta)=0$ and that $u$ is a weak solution. We first proceed by tacitly assuming that the total variation of all component of $u_{r}$ is uniformly bounded and, next, explain how to handle $U_{r}$ which is only in $L^{\infty}$. Denote by $\chi_{\operatorname{supp} \theta}^{k_{0}, h_{0}}$ the characteristic function of the support of the function $\theta$. We can write

$$
\begin{aligned}
\Delta\left(u_{r}, \theta\right) & =\sum_{k_{0}} \sum_{h_{0} \text { odd }} \int_{k_{0} s}^{\left(k_{0}+1\right) s} \int_{\left(k_{0}-1\right) r}^{\left(k_{0}+1\right) r}\left(u_{r} \partial_{t} \theta+f\left(u_{r}\right) \partial_{x} \theta+g\left(u_{r}\right) \theta\right) \mathrm{d} x \mathrm{~d} t \\
& =\Omega_{r}^{1}+\Omega_{r}^{2}+\Omega_{r}^{3}
\end{aligned}
$$

where

$$
\begin{gathered}
\Omega_{r}^{1}=\sum_{k_{0}} \sum_{h_{0} \text { odd }} O(1)\left(s^{2}+r^{2}\right)\left(s+r+\left|u_{k_{0}, h_{0}+1}-u_{k_{0}, h_{0}-1}\right|\right) \chi_{\operatorname{supp} \theta}^{k_{0}, h_{0}} \\
\Omega_{r}^{2}=\sum_{k_{0}} \sum_{h_{0} \text { odd }}\left(\int_{\left(k_{0}-1\right) r}^{\left(k_{0}+1\right) r} u_{r}\left(\left(k_{0}+1\right) s-, x\right) \theta\left(\left(k_{0}+1\right) s, x\right) \mathrm{d} x\right. \\
\left.-\int_{\left(k_{0}-1\right) r}^{\left(k_{0}+1\right) r} u_{r}\left(k_{0} s+, x\right) \theta\left(k_{0} s, x\right) \mathrm{d} x\right)
\end{gathered}
$$




$$
\begin{aligned}
\Omega_{r}^{3}=\sum_{k_{0}} \sum_{h_{0} \text { odd }} & \left\{\int_{k_{0} s}^{\left(k_{0}+1\right) s} f\left(u_{r}\left(t,\left(h_{0}+1\right) r-0\right)\right) \theta\left(t,\left(h_{0}+1\right) r\right) \mathrm{d} t\right. \\
& \left.-\int_{k_{0} s}^{\left(k_{0}+1\right) s} f\left(u_{r}\left(t,\left(h_{0}-1\right) r+0\right)\right) \theta\left(t,\left(h_{0}-1\right) r\right) \mathrm{d} t\right\} .
\end{aligned}
$$

Since $V_{r}, W_{r}$ have uniformly bounded total variation and $\theta$ is a smooth function one finds easily

$$
\Omega_{r}^{1}=O(1) r
$$

The term

$$
\Omega_{r}^{2}=-\sum_{k} \int_{-\infty}^{+\infty}\left[u_{r}\right](k s, x) \theta(k s, x) \mathrm{d} x
$$

can be rewritten in the form

$$
\Omega_{r}^{2}=\sum_{k} \sum_{h} \int_{(h-1) r}^{(h+1) r}\left(u_{r}\left(k s-,\left(h+a_{k}\right) r-\right)-u_{r}(k s-, h r-)\right) \theta(k s, x) \mathrm{d} x .
$$

This term was studied by Glimm [5] and, for almost every random sequence $\left(a_{k}\right)_{k}$, satisfies

$$
\left|\Omega_{r}^{2}\right| \leqslant C r
$$

In order to control $\Omega_{r}^{3}$, we use the Lipschitz continuity of the smooth function $f$ and obtain

$$
\begin{aligned}
\Omega_{r}^{3} & =\sum_{k_{0}} \sum_{h_{0} \text { odd }} \int_{k_{0} s}^{\left(k_{0}+1\right) s}\left(f\left(u_{r}\left(t,\left(h_{0}+1\right) r-\right)\right)-f\left(u_{r}\left(t,\left(h_{0}+1\right) r+0\right)\right)\right) \theta\left(t,\left(h_{0}+1\right) r\right) \mathrm{d} t \\
& =O(1) \sum_{k_{0}} \sum_{h_{0} \text { odd }} \int_{k_{0} s}^{\left(k_{0}+1\right) s}\left|u_{r}\left(t,\left(h_{0}+1\right) r+\right)-u_{r}\left(t,\left(h_{0}+1\right) r-\right)\right| \mathrm{d} t \chi_{\text {supp } \theta}^{k_{0}, h_{0}} \\
& \left.=O(1) \sum_{k_{0}} \sum_{h_{0} \text { odd }} \int_{k_{0} s}^{\left(k_{0}+1\right) s} t \mid \widetilde{u}_{k_{0}, h_{0}+1}\right)-g\left(\widetilde{u}_{k_{0}, h_{0}+1}\right) \mid \mathrm{d} t \chi_{\text {supp } \theta}^{k_{0}, h_{0}} \\
& =O(1) \sum_{k_{0}} \sum_{h_{0}} \int_{k_{0} s}^{\left(k_{0}+1\right) s} \operatorname{trd} \mathrm{d} \chi_{\text {supp } \theta}^{k_{0}, h_{0}},
\end{aligned}
$$

thus

$$
\Omega_{r}^{3}=O(1) r .
$$

Finally, we discuss the component $U_{r}$ which is only bounded. The original proof of Glimm requires that the approximate solution is of uniformly bounded total variation, but can be extended to our situation where the component $U_{r}$ is solely bounded. The main observation is that (8.32) must be replaced by the weaker statement

$$
\Omega_{r}^{2} \rightarrow 0 \quad \text { when } \quad r \rightarrow 0,
$$

which is clearly sufficient to deduce the result.

In conclusion we have

$$
\Delta\left(u_{r}, \theta\right) \rightarrow 0
$$

so that the limit function $u$ satisfies

$$
\Delta(u, \theta)=0
$$

and, therefore, is a weak solution of (8.1). The proof of theorem 8.7 is complete. 
Remark 8.8. It would be interesting to estimate the $L^{\infty}$ norm of the geometric variables $W$ from the initial data of the problem in order to make the condition (8.21) more explicit. In particular, this would require to be able to estimate the time of blow up from the initial data of the problem.

\section{Implications for numerical relativity}

In recent years numerical relativists have started to treat the special relativistic Euler equations using modern high resolution shock-capturing (HRSC) methods. There are many such algorithms, and a detailed comparison can be found in e.g. [8]. They involve three components, a reconstruction process, an (approximate or exact) Riemann solver and a time discretization method. Here we will use a third order, weighted essentially non-oscillatory (WENO) reconstruction [17], the (approximate) Marquina solver [3] and the third order total variation diminishing Runge-Kutta method (TVDRK3) advocated in [17], for we have found them to be robust and reliable even in extreme situations [9].

Note that the relativistic Euler equations (3.18) involve only first derivatives of $a$ and $b$. Thus we can form a closed system of evolution equations by adjoining the equations (5.6) for the $W$-variables. We call this the small system. Thus the simplest approach is to apply the HRSC algorithm to the Euler equations and some standard approach, e.g., iterated CrankNicholson to the W-equations. However this turns out to be inaccurate and significant violations of the constraint equations (3.9) and (3.10) develop. This is not surprising for those equations imply discontinuities in $b_{t x}$ and $b_{x x}$ which the standard approach cannot handle. Our next approach was to treat the first derivatives on an equal footing to the fluid variables and to apply WENO, Marquina and TVDRK3 schemes to the nine-dimensional system of equations. This reduced but did not eliminate the constraint violations.

Another approach is to integrate the complete system of $U$-variables (second derivatives), $V$-variables (fluid quantities) and $W$-variables described in section 4. Because this system has dimension 15 we call it the big system. Constraint violations, away from discontinuities, were reduced to acceptable limits. However the evolution of the $V$ and $W$ variables was identical to that of the small system! The $U$ variables were evolved and satisfied the constraint equations, but they were not coupled to the other variables.

The numerical results were improved when some coupling was enforced, as now described. Recall two of the $W$ equations (5.6)

$$
W_{4 t}-W_{5 x}=\cdots, \quad W_{5 t}-W_{4 x}=0,
$$

where the source term is irrelevant here. These say

$$
\left(b_{t}\right)_{t}-\left(b_{x}\right)_{x}=\cdots, \quad\left(b_{x}\right)_{t}-\left(b_{t}\right)_{x}=0 .
$$

We now replace $\left(b_{x}\right)_{x}$ by $b_{x x}=U_{4}$ and $\left(b_{t}\right)_{x}=b_{t x}=U_{3}$, i.e., we evolve

$$
W_{4 t}=U_{4}+\cdots, \quad W_{5 t}=U_{3} .
$$

The other two pairs of equations are treated similarly. This does not reduce the dimension of the system, it is still 15 , but the characteristic structure is considerably simplified. We call this the medium system.

In order to use characteristic WENO decomposition and the Marquina solver we need the eigenvalues of the $15 \times 15$ Jacobian matrix and left and right eigenvectors normalized by $\mathbf{l} \cdot \mathbf{r}=1$. In what follows we write right eigenvectors as vectors and left eigenvectors as covectors. Further $c_{ \pm}=1 \pm c_{s}{ }^{2}$ and $\lambda_{ \pm}=\left(v \pm c_{s}\right) /\left(1 \pm v c_{s}\right)$. Finally define

$$
D=\frac{v^{2}-c_{s}{ }^{2}}{4 c_{s}\left(1-v^{2}\right)} \text {. }
$$


Energy density and velocity at $t=0.5$

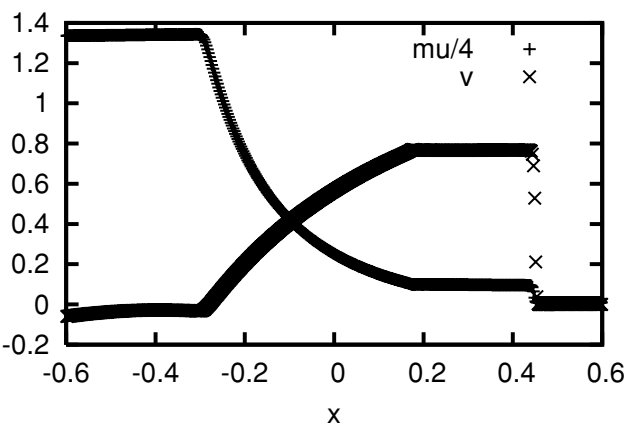

btx and bxx at $t=0.5$

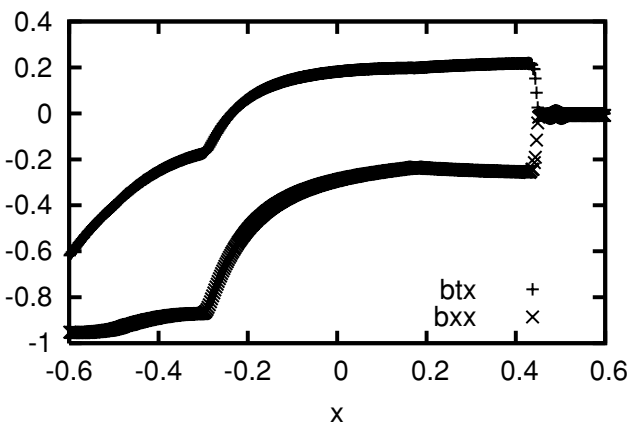

atx and $\mathrm{axx}$ at $\mathrm{t}=0.5$

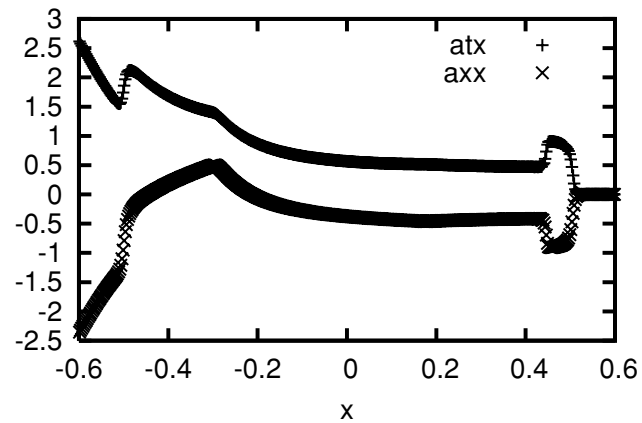

Constraints at $\mathrm{t}=0.5$

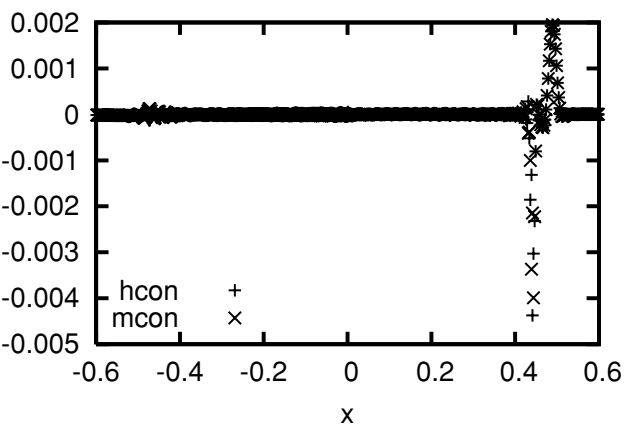

Figure 2. The shock tube evolution at time $t=0.5$. The four graphs show, as functions of $x$, respectively $\mu / 4$ and $v, a_{t x}$ and $a_{x x}, b_{t x}$ and $b_{x x}$, and finally the Hamiltonian and momentum constraints.

There is an eigenvalue $\lambda=0$ with multiplicity 7 . A basis of normalized eigenvectors is

$$
\begin{array}{llll}
l_{0}=\mathrm{d}\left(\mathrm{e}^{2 a}\right), & r_{0}=\partial / \partial \mathrm{e}^{2 a}+\frac{1}{2} \kappa \mu\left(\partial / \partial a_{x x}-\partial / \partial b_{x x}\right), \\
l_{1}=\mathrm{d}\left(a_{t}\right), & r_{1}=\partial / \partial a_{t}, & l_{2}=\mathrm{d}\left(a_{x}\right), & r_{2}=\partial / \partial a_{x}, \\
l_{3}=\mathrm{d}\left(b_{t}\right), & r_{3}=\partial / \partial b_{t}, & l_{4}=\mathrm{d}\left(b_{x}\right), & r_{4}=\partial / \partial b_{x}, \\
l_{5}=\mathrm{d}\left(c_{t}\right), & r_{5}=\partial / \partial c_{t}, & l_{6}=\mathrm{d}\left(c_{x}\right), & r_{6}=\partial / \partial c_{x} .
\end{array}
$$

The eigenvalue $\lambda=-1$ has multiplicity 3 and the eigenspace has a normalized basis $l_{7}=\frac{1}{4} \kappa \mu c_{d}\left(\mathrm{e}^{2 a}\right)+\frac{1}{2} d\left(b_{t x}+b_{x x}\right)+\frac{1}{4} \kappa \mathrm{e}^{2 a} d(\tau-S)$,

$$
\begin{array}{llrl}
l_{7} & =\frac{1}{4} \kappa \mu c_{d}\left(\mathrm{e}^{2 a}\right)+\frac{1}{2} d\left(b_{t x}+b_{x x}\right)+\frac{1}{4} \kappa \mathrm{e}^{2 a} d(\tau-S), & r_{7} & =\partial / \partial b_{t x}+\partial / \partial b_{x x}, \\
l_{8} & =-\frac{1}{4} \kappa \mu c_{+} d\left(\mathrm{e}^{2 a}\right)+\frac{1}{2} d\left(a_{t x}+a_{x x}\right)-\frac{1}{4} \kappa \mathrm{e}^{2 a}\left(c_{+} / c_{-}\right) d(\tau-S), & r_{8} & =\partial / \partial a_{t x}+\partial / \partial a_{x x}, \\
l_{9} & =\frac{1}{2} d\left(c_{t x}+c_{x x}\right), & r_{9} & =\partial / \partial c_{t x}+\partial / \partial c_{x x} .
\end{array}
$$$$
l_{9}=\frac{1}{2} d\left(c_{t x}+c_{x x}\right) \text {, }
$$

The eigenvalue $\lambda=1$ has multiplicity 3 and the eigenspace has a normalized basis

$l_{10}=-\frac{1}{4} \kappa \mu c_{d}\left(\mathrm{e}^{2 a}\right)+\frac{1}{2} d\left(b_{t x}-b_{x x}\right)-\frac{1}{4} \kappa \mathrm{e}^{2 a} d(\tau+S), \quad r_{10}=\partial / \partial b_{t x}-\partial / \partial b_{x x}$,

$l_{11}=\frac{1}{4} \kappa \mu c_{+} d\left(\mathrm{e}^{2 a}\right)+\frac{1}{2} d\left(a_{t x}-a_{x x}\right)-\frac{1}{4} \kappa \mathrm{e}^{2 a}\left(c_{+} / c_{-}\right) d(\tau+S), \quad r_{11}=\partial / \partial a_{t x}-\partial / \partial a_{x x}$,

$l_{12}=\frac{1}{2} d\left(c_{t x}-c_{x x}\right)$,

$r_{12}=\partial / \partial c_{t x}-\partial / \partial c_{x x}$.

The eigenvalue $\lambda=\lambda_{-}$is simple with eigenvectors

$l_{13}=-\left(c_{+} / c_{-}\right) D\left(-\mathrm{d} \tau+(\mathrm{d} S) / \lambda_{+}\right)$

$r_{13}=\kappa \mathrm{e}^{2 a}\left[\partial / \partial a_{t x}-\lambda_{-}{ }^{-1} \partial / \partial a_{x x}-\left(c_{-} / c_{+}\right)\left(\partial / \partial b_{t x}-\lambda_{-}{ }^{-1} \partial / \partial b_{x x}+2 \lambda_{-}{ }^{-1} \partial / \partial \tau+2 \partial / \partial S\right)\right]$, 
Energy density and velocity at $\mathrm{t}=0.5$

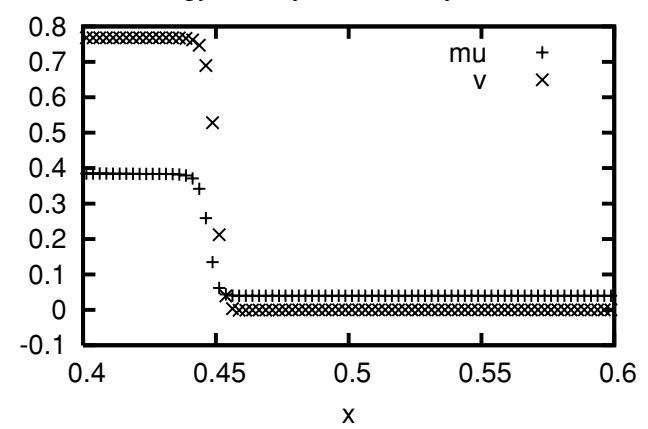

btx and bxx at $t=0.5$

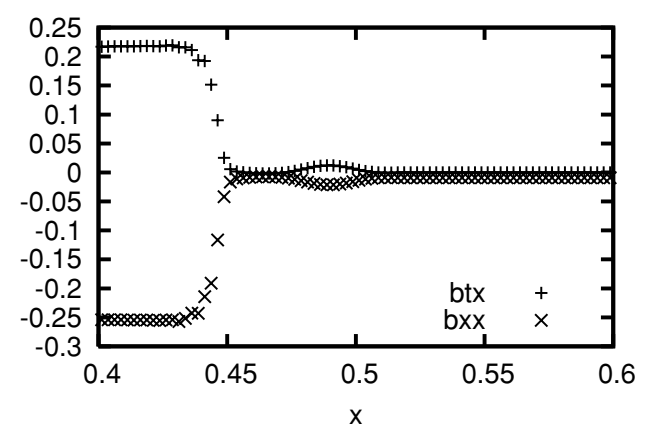

atx and axx at $t=0.5$

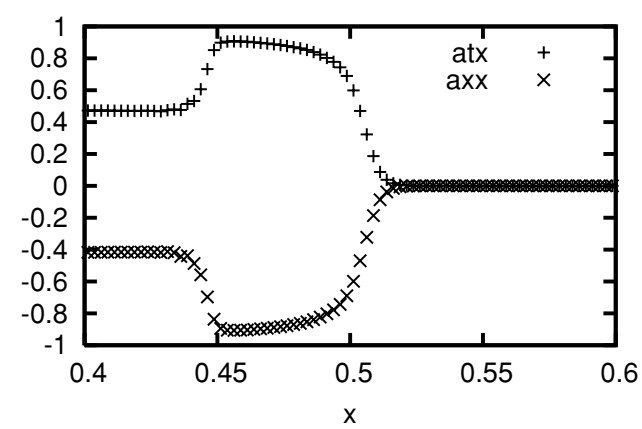

Constraints at $t=0.5$

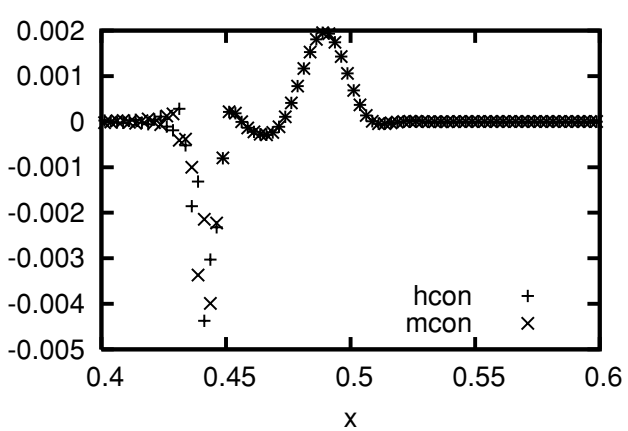

Figure 3. The shock tube evolution at time $t=0.5$. The four graphs show, as functions of $x$, respectively $\mu / 4$ and $v, a_{t x}$ and $a_{x x}, b_{t x}$ and $b_{x x}$, and finally the Hamiltonian and momentum constraints. This is a 'zoom-in' of figure 2 to the range $0.4 \leqslant x \leqslant 0.5$.

and the eigenvalue $\lambda=\lambda_{+}$is simple with eigenvectors

$l_{14}=-\left(c_{+} / c_{-}\right) D\left(\mathrm{~d} \tau-(\mathrm{d} S) / \lambda_{-}\right)$,

$r_{14}=\kappa \mathrm{e}^{2 a}\left[\partial / \partial a_{t x}-\lambda_{+}{ }^{-1} \partial / \partial a_{x x}-\left(c_{-} / c_{+}\right)\left(\partial / \partial b_{t x}-\lambda_{+}{ }^{-1} \partial / \partial b_{x x}+2 \lambda_{+}{ }^{-1} \partial / \partial \tau+2 \partial / \partial S\right)\right]$.

We now describe two numerical simulations in which $c(t, x) \equiv 0$. The first simulation is a general relativistic version of the classic shock tube problem [20]. We chose time-symmetric initial data with $v(0, x)=S(0, x)=a_{t}(0, x)=b_{t}(0, x)=0$. We also chose $a(0, x)=-2$. The constraint equation (3.10) is identically satisfied. We chose

$$
\kappa \mu(0, x)= \begin{cases}100, & x<0, \\ 1, & x>0,\end{cases}
$$

and $c_{s}{ }^{2}=1 / 3$ appropriate to black-body radiation. The remaining constraint equation (3.9) is now a first-order ordinary differential equation for $b_{x}$ which can be solved analytically for $x<0$ and $x>0$. Setting $f=\kappa \mathrm{e}^{2 a} \tau$, a constant, we have

$$
b_{x}=-\sqrt{\frac{f}{3}} \tan \left(\frac{1}{2} \sqrt{3 f} x+C\right),
$$

where $C$ is an integration constant. The two constants of integration were used to make $b_{x}$ continuous at $x=0$ and to place the curvature singularity well away from the domain of integration $-1 \leqslant x \leqslant 1$. Our cell-centred simulation used 800 cells with a Courant number of 0.5 . 
Energy density and velocity at $\mathrm{t}=0.3$

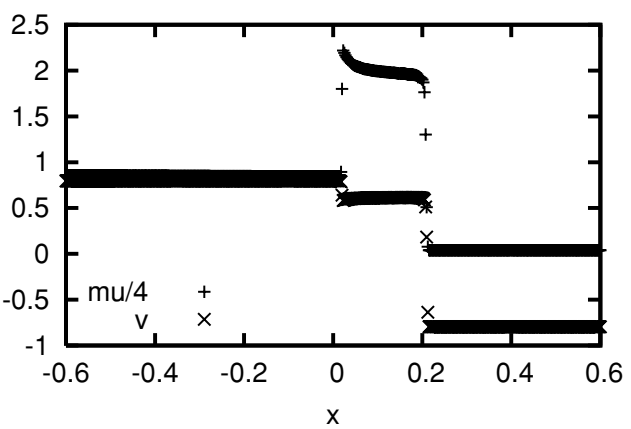

btx and bxx at $t=0.3$

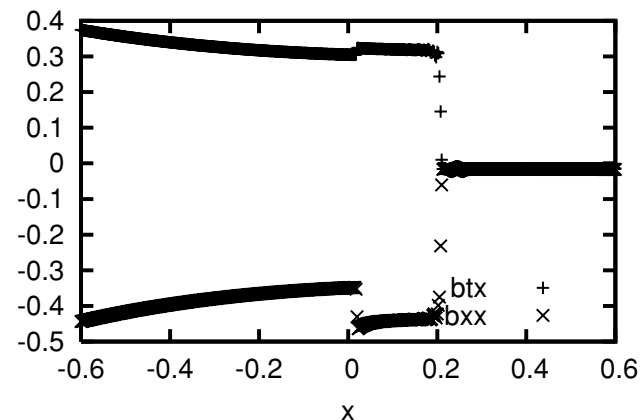

atx and axx at $\mathrm{t}=0.3$

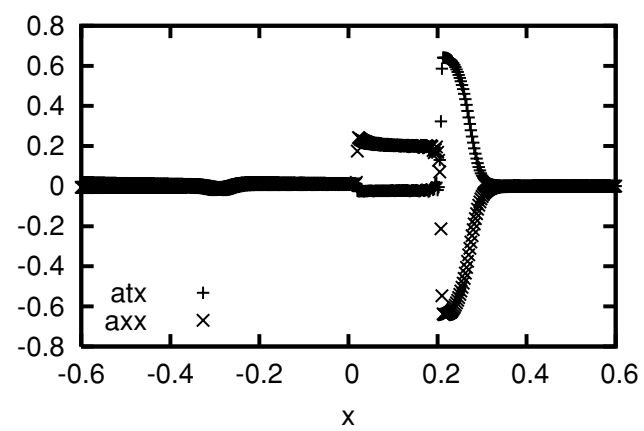

Constraints at $t=0.3$

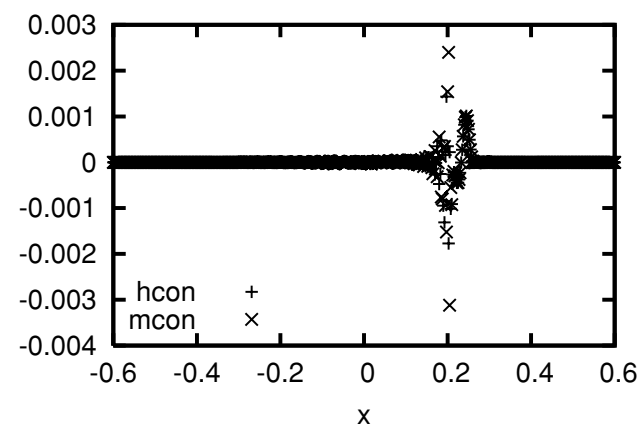

Figure 4. The collision evolution at time $t=0.3$. The four graphs show, as functions of $x$, respectively $\mu / 4$ and $v, a_{t x}$ and $a_{x x}, b_{t x}$ and $b_{x x}$, and finally the Hamiltonian and momentum constraints.

Figure 2 shows as functions of $x$, respectively $\mu / 4$ and $v, a_{t x}$ and $a_{x x}, b_{t x}$ and $b_{x x}$ and finally the Hamiltonian and momentum constraints at time $t=0.5$. Figure 3 is a 'zoom-in' of figure 2 covering a much smaller $x$-range.

There is a right moving hydrodynamical shock which has reached $x \approx 0.45$ from $x=0$. We know from the constraint equations and the evolution equations for the second derivatives that discontinuities in the second derivatives should appear at the same point, and these are clearly visible in figure 3 . For the geometry variables we also expect effects travelling at velocity \pm 1 , which should be visible at $x= \pm 0.5$. Indeed there are discontinuities in the second derivatives of $a$ at these two $x$-values, although in our simulation they are not as well resolved as the primary one at $x=0.45$. The constraint equations require discontinuities in the second derivatives of $b$ at hydrodynamic shocks but prohibit them elsewhere, and so there are no jumps in $b_{t x}$ or $b_{x x}$ at $x= \pm 0.5$. Away from discontinuities the constraints are as low as one would expect from a continuous simulation, and this has been verified by convergence tests.

The second simulation, the collision problem, is similar to the first. There are again two density states but the transition between them is initially continuous and the states are moving towards each other

$$
\mu(0, x)=\frac{40}{\kappa}(1.1+\tanh (-40 x)), \quad v(0, x)=\frac{20}{\kappa} \tanh (-40 x) .
$$

We chose $a(0, x)=-3.0$ and $a_{t}(0, x)=0$. We treated the constraint equations as coupled ordinary differential equations for $b_{t}(0, x)$ and $b_{x}(0, x)$ and solved them numerically assuming they both vanished at $x=0$. 
Energy density and velocity at $t=0.3$

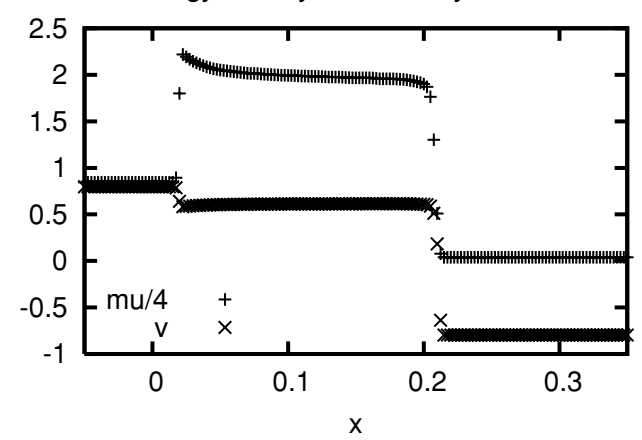

btx and $b x x$ at $t=0.3$

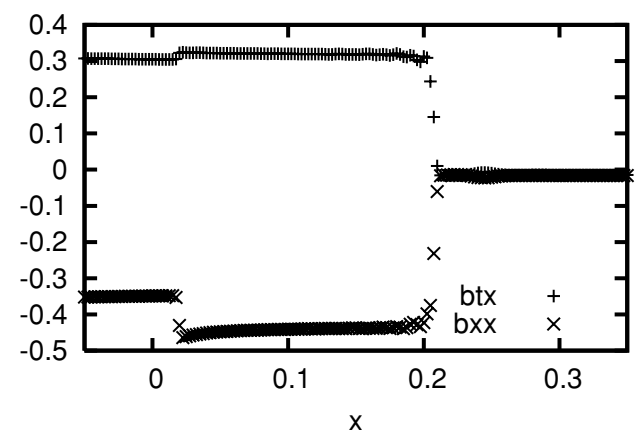

atx and $\operatorname{axx}$ at $\mathrm{t}=0.3$

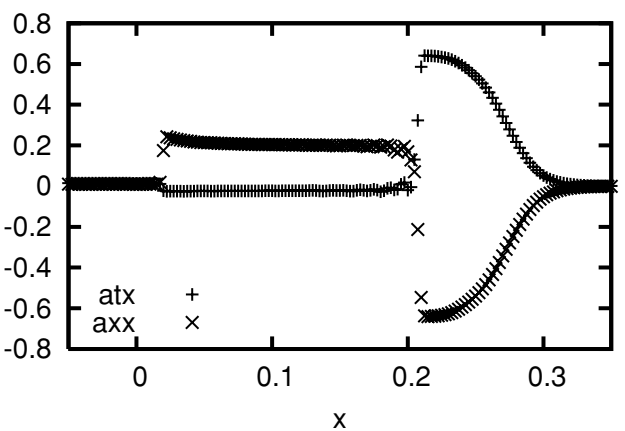

Constraints at $\mathrm{t}=0.3$

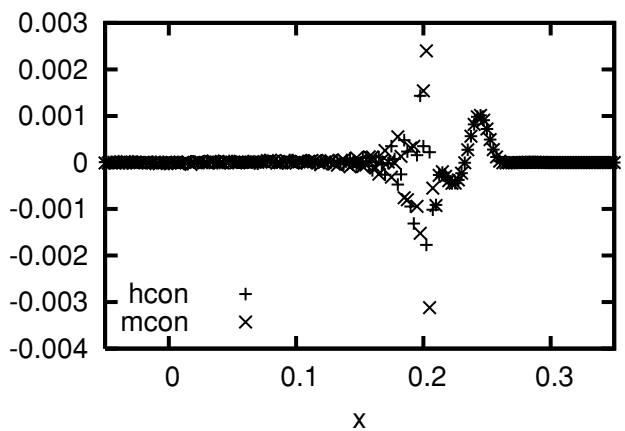

Figure 5. The collision evolution at time $t=0.3$. The four graphs show, as functions of $x$, respectively $\mu / 4$ and $v, a_{t x}$ and $a_{x x}, b_{t x}$ and $b_{x x}$, and finally the Hamiltonian and momentum constraints. This is a 'zoom-in' of figure 4 to the range $-0.05 \leqslant x \leqslant 0.35$.

We describe the solution at $t=-0.3$, and figures 4 and 5 are the analogues of figures 2 and 3. Although the hydrodynamical variables were initially smooth, two shocks have developed at $x \approx 0.02$ where the density more than doubles and at $x \approx 0.22$ where it decreases by a factor of about 40 . The $a$-derivatives have a small blip at $x=-0.3$ travelling to the left at the speed of light. There are two shocks corresponding to the hydrodynamic shocks, and a rarefaction fan for $0.22 \leqslant x \leqslant 0.3$. The $b$-derivatives merely have shocks corresponding to the hydrodynamical ones. Because the shock at $x \approx 0.02$ is relatively weak the constraints are satisfied to satisfactory precision there. There are still $O(\Delta x)$ deviations at the strong shock. However this feature originated much earlier and has been moving to the right. As it does so the constraint violations in its wake can be seen to reduce quickly to satisfactory levels. This is good news for numerical relativity.

\section{Conclusions}

At first sight it might appear incongruous, given the title of this paper, to discuss the propagation of discontinuities in the curvature tensor of vacuum spacetimes. However this topic, discussed in section 2, is the key to our results, for it forces us to look at the constraint equations. If a discontinuity arises it must satisfy the constraint equations on any Cauchy surface, and so can occur only in certain second derivatives of the trace-free part of the spatial 3-metric. In this case we were able to produce a fairly general existence/uniqueness theorem. 
We are primarily concerned with curvature discontinuities produced during the evolution of an inviscid fluid, and cannot expect to produce existence results which are more general than those where self-gravity is not included, and so we have restricted consideration to spacetimes with planar symmetry, and in particular polarized Gowdy spacetimes. Our main existence result was stated in section 6 , and the formal proof was outlined in the next two sections. Some exploratory numerical calculations are reported in section 9 . Here we see explicitly that where there are hydrodynamic shocks, certain second derivatives of the metric components $\left(b_{t x}\right.$ and $b_{x x}$ ) also have shocks, as required for the constraints to be fulfilled. Other second derivatives $\left(a_{t x}\right.$ and $\left.a_{x x}\right)$ contain shocks or rarefaction fans propagating at the speed of light, as predicted by our theory.

All of this work is in progress, and suggests many new questions. One outstanding theoretical question is how do matter shock waves interact/affect spacetime singularities? Another is that, given that we now understand the nature of the problem, can we improve on the quality of the numerical calculations reported in section 9? Preliminary results, to be reported, suggest that we can.

\section{Acknowledgments}

APB was supported by a New Zealand Bright Future Scholarship. PGL and JMS acknowledge hospitality provided by the Isaac Newton Institute of Mathematical Sciences, Cambridge during the Programme on Nonlinear Hyperbolic Waves in Phase Dynamics and Astrophysics (January-July 2003). JMS thanks the Max Planck Institute for Gravitational Physics (Albert Einstein Institute), Golm, Germany for their generous unstinting support.

\section{References}

[1] Anguige K 2000 A class of plane symmetric perfect-fluid cosmologies with a Kasner-like singularity Class. Quantum Grav. 17 2117-28

[2] Christodoulou D 1992 Bounded variation solutions of the spherically symmetric Einstein-scalar field equations Commun. Pure Appl. Math. 46 1131-20

[3] Donat R, Font J A, Ibáñez J M and Marquina A 1998 A flux-split algorithm applied to relativistic flows J. Comp. Phys. 146 58-81

[4] van Elst H, Ellis G F R and Schmidt B G 2000 Propagation of jump discontinuities in relativistic cosmology Phys. Rev. D 62104023

[5] Glimm J 1965 Solutions in the large for nonlinear hyperbolic systems of equations Commun. Pure Appl. Math. 18 697-715

[6] Groah J M and Temple B 2004 Shock wave solutions of the Einstein equations with perfect fluid sources: existence and consistency by a locally inertial Glimm Scheme Memoirs of the AMS at press

[7] Groah J M and Temple B 2002 A locally inertial Glimm scheme for general relativity Math. Contemp. 22 163-79

[8] Hawke I 2001 Computational ultrarelativistic hydrodynamics PhD Dissertation University of Cambridge

[9] Hawke I and Stewart J M 2002 The dynamics of primordial black-hole formation Class. Quantum Grav. 19 3687-707

[10] LeFloch P G 1990 Glimm method applied to quasilinear hyperbolic systems with source term Habilitation à Diriger des Recherches Université Pierre et Marie Curie (Paris VI) chapter 11

[11] LeFloch P G 2002 Hyperbolic systems of conservation laws: the theory of classical and nonclassical shock waves Lectures in Mathematics, ETH Zürich (Basle: Birkhäuser)

[12] Lichnerowicz A 1993 Magnetohydrodynamics: Waves and Shock Waves in Curved Spacetime (Dordrecht: Kluwer) vol 14

[13] Nishida T 1968 Global solution for an initial boundary value problem of a quasilinear hyperbolic system Proc. Japan Acad. Sci. 44 642-6 
[14] Nishida T and Smoller J A 1973 Solutions in the large for some nonlinear hyperbolic conservation laws Commun. Pure Appl. Math. 26 183-200

[15] Rendall AD 1990 Reduction of the characteristic initial value problem to the Cauchy problem and its applications to the Einstein equations Proc. R. Soc. Lond. A 427 221-39

[16] Rendall A D 2000 Blow-up for solutions of hyperbolic PDE and spacetime singularities Journées Equations aux Dérivées Partielles (Nantes, June 2000, GRD 1151 (CNRS) France) (Preprint gr-qc 0006060)

[17] Shu C-W 1991 High order ENO and WENO schemes for computational fluid dynamics High-Order Methods for Computational Physics ed T J Barth and H Deconinck (Berlin: Springer) pp 439-582

[18] Smoller J A and Temple B 1993 Global solutions of the relativistic Euler equations Commun. Math. Phys. 156 67-99

[19] Taub A H 1957 Approximate solutions of the Einstein equations for isentropic motions of plane-symmetric distributions of perfect fluids Phys. Rev. $107884-900$

[20] Thompson K 1986 The special relativistic shock tube J. Fluid Mech. 171 365-75

[21] Wald R M 1984 General Relativity (Chicago, IL: Universty of Chicago Press) 